\title{
Curves in $\mathbf{P}^{3}$ Whose Ideals Are Simple in a Certain Numerical Sense
}

\author{
By
}

\author{
Mutsumi AMASAKI*
}

\section{Introduction}

For a curve $X$ in $\mathbb{P}^{3}$ (see Notation), we define the basic sequence of $X$ to be the sequence of positive integers $\left(a ; n_{1}, \ldots, n_{a} ; n_{a+1}, \ldots, n_{a+b}\right)$ $(b \geq 0)$ which satisfies the three conditions described below, and denote it by $B(X)$ (see $[2 ; \S 1])$.

(I. 1) $a \leq n_{1} \leq n_{2} \leq \cdots \leq n_{a}, n_{1} \leq n_{a+1} \leq n_{a+2} \leq \cdots \leq n_{a+b}$, where $\left(n_{a+1}, \ldots\right.$, $\left.n_{a+b}\right)$ is empty if $b=0$.

(I.2) Let $\mathscr{I}_{X}$ denote the ideal sheaf of $X$ and set

$$
\left(\begin{array}{l}
n \\
m
\end{array}\right)_{+}=\left\{\begin{array}{cl}
\frac{n !}{(n-m) ! m !} & \text { if } n \geq m \geq 0 \\
0 & \text { otherwise }
\end{array}\right. \text {. }
$$

Then

$$
h^{0}\left(\mathscr{I}_{X}(n)\right)=\left(\begin{array}{c}
n-a+3 \\
3
\end{array}\right)_{+}+\sum_{i=1}^{a}\left(\begin{array}{c}
n-n_{i}+2 \\
2
\end{array}\right)_{+}+\sum_{j=1}^{b}\left(\begin{array}{c}
n-n_{a+j}+1 \\
1
\end{array}\right)_{+} .
$$

(I.3) Let $L$ be a generic line in $\mathbb{P}^{3}$ not meeting $X$. Then

$$
\operatorname{dim}_{k} \operatorname{Im}\left(\mathrm{H}^{0}\left(\mathscr{I}_{X}(n)\right) \stackrel{\text { rest }}{\longrightarrow} \mathrm{H}^{0}\left(\left.\mathscr{I}_{X}(n)\right|_{L}\right)\right)=\left(\begin{array}{c}
n-a+1 \\
1
\end{array}\right)_{+}+\sum_{i=1}^{a}\left(\begin{array}{c}
n-n_{i} \\
0
\end{array}\right)_{+},
$$

where rest denotes the restriction map.

The condition (I.3) may be exchanged for the following one;

Communicated by M. Kashiwara, May 2, 1987. Revised July 29, 1987.

* Research Institute for Mathematical Sciences, Kyoto University, Kyoto 606, Japan. 
(I. 3)' Let $x_{1}, x_{2}, x_{3}, x_{4}$ be the homogeneous coordinates of $\mathbf{P}^{3}$ chosen sufficiently generally. Then the deficiency module $M(X):=\underset{n}{\oplus} \mathrm{H}^{1}\left(\mathscr{I}_{X}(n)\right)$ has a minimal free resolution of the form

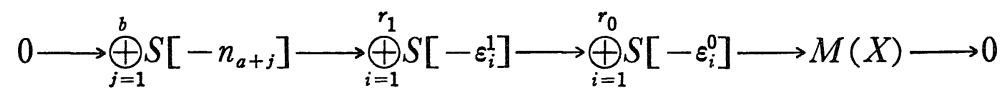

as an $S$-module, where $S=k\left[x_{3}, x_{4}\right]$ and $\varepsilon_{i}^{j}\left(1 \leq i \leq r_{j}, j=0,1\right)$ are integers (see $[2 ; \S 2]$ ).

Generally, it is not easy to determine the basic sequence of an arbitrary curve or to decide whether or not a given basic sequence actually corresponds to a curve of some good nature (smooth, irreducible etc.). In this paper we treat the second problem in the case where $B:=B(X)$ takes the simplest form, $a \leq n_{1} \leq \cdots \leq n_{a} \leq n_{a+1} \leq$ $\cdots \leq n_{a+b} \leq a+1$, and prove an existence theorem for smooth irreducible curves with such basic sequences. When $B$ fulfills this condition, we say that $B$ is neat.

Theorem (I. 4). A neat basic sequence $B$ is realised by a smooth irreducible curve in $\mathbf{P}^{3}$ such that the cohomology of its normal bundle vanishes in degree one, if $B$ satisfies one of the following conditions.

1) $B=\left(a ; a^{a} ; a^{b}\right)$ with $0 \leq b<\rho(a)$,

2) $B=\left(a ;(a+1)^{a} ;(a+1)^{b}\right)$ with $0 \leq b<\rho(a+1)$,

3) $B=\left(a ; a^{a} ;(a+1)^{b}\right)$ with $0 \leq b \leq \rho(a-1)$,

4) $B=\left(a ; a^{c},(a+1)^{a-c} ;(a+1)^{b}\right)$ with $1 \leq c \leq a-1,0 \leq b \leq a-2$,

5) $B=\left(a ; a^{a} ; a^{c},(a+1)^{b-c}\right)$ with $1 \leq c \leq b-1 \leq a-3$, where $\rho(n)=[n / 2](n-[n / 2])$ and $[x]$ denotes the integral part of $x$.

A curve with neat basic sequence is a curve of maximal rank (see (1.11)), so it is natural to expect that the basic sequences of the curves given by Ballico-Ellia in [5] are neat. This is verified at least in two cases; in particular the cases $\left(a ; a^{a} ; a^{b}\right)$ with

$$
\frac{(a-3)(a+2)}{3} \leq b \leq \frac{(a-2)(2 a+3)}{6}
$$

and $\left(a ;(a+1)^{a} ;(a+1)^{b}\right)$ with

$$
\frac{(a-2)\left(a^{2}+2 a-6\right)}{3(a-1)} \leq b \leq \frac{(a-1)\left(2 a^{2}+5 a-6\right)}{6 a}
$$


correspond to smooth irreducible curves (see (1.12)). On the other hand, by the works on projectively Cohen-Macaulay curves [9] and arithmetically Buchsbaum curves [1], [2], [3] and [7; §5], we know a lot of smooth irreducible curves having neat basic sequences which satisfy $0 \leq b \leq a / 2$ (see (1.13)). In view of these facts the theorem above has been worked out with the aim of widening the known range of existence. The result, however, gives only a partial answer and the problem still remains open.

In each case, the proof of the theorem consists of two steps. In the first step, we construct a reduced singular curve which has a given neat basic sequence and whose support is a connected union of lines, starting with a projectively Cohen-Macaulay singular curve $X_{0}$ such that $B\left(X_{0}\right)=\left(a ; a^{a}\right)$ and then removing suitable lines from it. In the second step, with the help of the criterion for the smoothability of a singular curve due to Hartshorne-Hirschowitz [10] and Sernesi [12], it is shown that the curve obtained in the first step is flatly smoothable in $\mathbb{P}^{3}$. In our case, we need cumbersome computations of polynomials to verify the conditions in the criterion, for the geometric method as in [10] or [12] cannot be applied.

Each singular curve appearing in the course of the proof has only plane singularities, but their multiplicities are larger than two in most cases. If we prefer only nodes as singular points, we can deform the curve flatly in $\mathbb{P}^{3}$ over an irreducible parameter space so that it becomes a union of lines, no three of which meet at a point. This enables us to get new smoothable stick figures (see [10], (7.1) and (7.2)).

In this paper we do not at all discuss the fundamental but subtle problem whether or not integral curves with the same neat basic sequence form an irreducible open subscheme of $\mathrm{Hilb}\left(\mathbb{P}^{3}\right)$, because the answer is not obtained except in few simple cases where it is known to be affirmative.

\section{Notation}

The ground field $k$ is of characteristic zero and algebraically closed. We mean by the word curve an equidimensional complete scheme over $k$ of dimension one without any embedded points. The 
polynomial rings $k\left[x_{1}, x_{2}, x_{3}, x_{4}\right]$ and $k\left[x_{3}, x_{4}\right]$ are denoted by $R$ and $S$ respectively, where $x_{1}, x_{2}, x_{3}, x_{4}$ are the homogeneous coordinates of $\mathbf{P}^{3}$. The ideal sheaf of a curve $X$ in $\mathbb{P}^{3}$ is denoted by $\mathscr{I}_{X}$. We set $I_{X}=\oplus \mathrm{H}^{0}\left(\mathscr{I}_{X}(n)\right) \subset R, \quad M(X)=\oplus \mathrm{H}^{1}\left(\mathscr{I}_{X}(n)\right) \quad$ and $\quad M^{\prime}(X)=$ $\operatorname{Hom}_{k}(M(X), k)$. The notations of [2] and [3] will be used freely.

\section{§1. Numerical Properties and Known Examples}

Let $B=\left(a ; n_{1}, \ldots, n_{a} ; n_{a+1}, \ldots, n_{a+b}\right) \quad(a \geq 1, b \geq 0)$ be a sequence of positive integers. We will call its three sections divided by semicolons the head, the body and the tail in order and refer to the subsequence $\left(a ; n_{1}, \ldots, n_{a}\right)$ as the principal part of $B$. The head is denoted by $a(B)$ and the number $b$, which we call the size of the tail, by $b(B)$. In the case $b(B)=0, B$ consists of the principal part only. We put

$$
\left\{\begin{array}{l}
\operatorname{deg}(B)=\sum_{i=1}^{a} n_{i}-\frac{1}{2} a(a-1)-b \\
\operatorname{gen}(B)=1-\frac{1}{6} a(a-1)(a-5)+\frac{1}{2} \sum_{i=1}^{a} n_{i}\left(n_{i}-3\right)-\sum_{j=1}^{b} n_{a+j}+b
\end{array}\right.
$$

and call $\operatorname{deg}(B)$ and $\operatorname{gen}(B)$ the degree and the genus of $B$ respectively. When $B$ is the basic sequence of a curve in $\mathbb{P}^{3}$, they coincide with its degree and arithmetic genus (cf. [2; Remark 1.9]).

Lemma (1.2). With the notation above, put $B^{\prime}=\left(a ; n_{1}, \ldots, n_{a-1}\right.$, $\left.n_{a}+1 ; n_{a+1}, \ldots, n_{a+b}\right)$ and $B^{\prime \prime}=\left(a ; n_{1}, \ldots, n_{a} ; n_{a+1}, \ldots, n_{a+b}, n\right)$ for $a$ positive integer $n$. Then

1) $\operatorname{deg}\left(B^{\prime}\right)=\operatorname{deg}(B)+1, \operatorname{gen}\left(B^{\prime}\right)=\operatorname{gen}(B)+n_{a}-1$.

2) $\operatorname{deg}\left(B^{\prime \prime}\right)=\operatorname{deg}(B)-1, \operatorname{gen}\left(B^{\prime \prime}\right)=\operatorname{gen}(B)-(n-1)$.

Definition (1.3). We say that a sequence of positive integers $\left(a ; n_{1}, \ldots, n_{a} ; n_{a+1}, \ldots, n_{a+b}\right) \quad(a \geq 1, b \geq 0)$ is neat or is a neat basic sequence if it satisfies the inequalities $a \leq n_{1} \leq \cdots \leq n_{a} \leq n_{a+1} \leq \cdots \leq n_{a+b} \leq$ $a+1$.

We begin by establishing some numerical properties of neat basic sequences. First, the formula (1.1) yields 
(1.4) $\left\{\begin{array}{l}\operatorname{deg}\left(a ; a^{m},(a+1)^{a-m}\right)=\frac{1}{2} a(a+1)+a-m \\ \operatorname{gen}\left(a ; a^{m},(a+1)^{a-m}\right)=\frac{1}{6}(a-1)(a-2)(2 a+3)+(a-1)(a-m)\end{array}\right.$

for $a \geq 1,0 \leq m \leq a$. Let $g$ be a nonnegative integer. Since

$$
\left\{\begin{array}{l}
\operatorname{gen}\left(a ; a^{m},(a+1)^{a-m}\right)=\operatorname{gen}\left(a ; a^{m+1},(a+1)^{a-m-1}\right)+a-1 \\
\operatorname{gen}\left(a+1 ;(a+1)^{a+1}\right)=\operatorname{gen}\left(a ;(a+1)^{a}\right)+a-1
\end{array}\right.
$$

(cf. 1) of (1.2)), the function gen $\left(a ; a^{m},(a+1)^{a-m}\right) \quad(a \geq 2,0 \leq m \leq a)$ increases strictly according as $m$ decreases or $a$ increases, with gen $\left(2 ; 2^{2}\right)=0$. There exist therefore integers $a, m, n$ satisfying $a \geq 2$, $0 \leq m \leq a, 0 \leq n<a-1$ such that

$$
g=\operatorname{gen}\left(a ; a^{m},(a+1)^{a-m}\right)+n,
$$

which are determined uniquely by this condition. With the use of these integers, we define the neat basic sequence $B_{\langle g\rangle}$ associated with $g$ to be

$$
\begin{cases}\left(a ; a^{m},(a+1)^{a-m}\right) & \text { if } n=0, \\ \left(a ; a^{m+n-a},(a+1)^{2 a-m-n} ;(a+1)^{a-n-1}\right) & \text { if } n \neq 0, m+n \geq a, \\ \left(a+1 ;(a+1)^{l+m+n+1},(a+2)^{a-l-m-n} ;(a+1)^{m-l+1},(a+2)^{a-m-n-1}\right) \\ & \text { if } n \neq 0, m+n<a,\end{cases}
$$

where $l=\min (a-m-n, m+1)$. It can be verified directly that

$$
\left\{\begin{array}{l}
\operatorname{gen}\left(B_{\langle g\rangle}\right)=g \\
0 \leq b\left(B_{\langle g\rangle}\right) \leq a-2
\end{array}\right. \text {. }
$$

Note that

$$
\operatorname{deg}\left(B_{\left\langle g_{0}+n\right\rangle}\right)=\operatorname{deg}\left(B_{\left\langle g_{0}\right\rangle}\right)+1
$$

for all $n, g_{0}=\operatorname{gen}\left(a ; a^{m},(a+1)^{a-m}\right)$ satisfying $a \geq 2,0 \leq m \leq a$ and $1 \leq n$ $\leq a-1$. Given a neat basic sequence $B$, we define next $(B)$ by the following rule;

1) in the case $B=\left(a ; a^{a} ; a^{m},(a+1)^{b-m}\right)$ with $0 \leq m \leq b$, set next $(B)$ $=\left(a ; a^{l},(a+1)^{a-l} ; a^{m-l},(a+1)^{a+b-m-1}\right)$, where $l=\min (a, m)$,

$2)$ in the case $B=\left(a ; a^{m},(a+1)^{a-m} ;(a+1)^{b}\right)$ with $0 \leq m<a, b \geq 0$, set $\operatorname{next}(B)=\left(a+1 ;(a+1)^{l+m+2},(a+2)^{a-l-m-1} ;(a+1)^{b-l+m},(a+2)^{a-m-1}\right)$, where $l=\min (b+m, a-m-1)$. 
Direct computations show that $\operatorname{deg}(\operatorname{next}(B))=\operatorname{deg}(B)+1$, gen $($ next $(B))=\operatorname{gen}(B)$.

Lemma (1.8). Let $B_{1}$ and $B_{2}$ be neat basic sequences.

1) $B_{1}=B_{2}$ if and only if $\operatorname{deg}\left(B_{1}\right)=\operatorname{deg}\left(B_{2}\right)$ and $\operatorname{gen}\left(B_{1}\right)=\operatorname{gen}\left(B_{2}\right)$.

2) If $\operatorname{deg}\left(B_{1}\right)>\operatorname{deg}\left(B_{2}\right)$, gen $\left(B_{1}\right)=\operatorname{gen}\left(B_{2}\right)$ and $a\left(B_{1}\right)=a\left(B_{2}\right)$, then $b\left(B_{1}\right)>a\left(B_{1}\right)-2$.

Proof. 1) For a sequence $B=\left(a ; n_{1}, \ldots, n_{a} ; n_{a+1}, \ldots, n_{a+b}\right)$ of positive integers, the polynomial

$$
K_{B}(x):=\left(\begin{array}{c}
x-a+3 \\
3
\end{array}\right)+\sum_{i=1}^{a}\left(\begin{array}{c}
x-n_{i}+2 \\
2
\end{array}\right)+\sum_{j=1}^{b}\left(\begin{array}{c}
x-n_{a+j}+1 \\
1
\end{array}\right)
$$

coincides with $\left(\begin{array}{c}x+3 \\ 3\end{array}\right)-(\operatorname{deg}(B) \cdot x+1-\operatorname{gen}(B))$ (cf. [2; Remark 1.9]), where $\left(\begin{array}{l}x \\ m\end{array}\right)=x(x-1) \cdots(x-m+1) / m ! \quad(m \geq 0)$. Suppose $B$ is neat. Since $\left(\begin{array}{l}x \\ m\end{array}\right)=\left(\begin{array}{l}x \\ m\end{array}\right)_{+}$for $x \geq m \geq 0$, we find that $K_{B}(a-1)=-\#\left\{j \mid n_{a+j}=\right.$ $a+1(1 \leq j \leq b)\}, K_{B}(a)=1+\#\left\{i \mid n_{i}=a(1 \leq i \leq a+b)\right\}, K_{B}(n)>0$ for $n \geq a$, and in particular $a=1+\max \left\{x \mid K_{B}(n) \leq 0, n \in Z\right\}$. The assertion follows from this immediately.

2) If $B_{1}$ and $B_{2}$ satisfy the hypothesis, we can apply next ( ) successively, starting with $B_{2}$, to get a neat basic sequence $B$ such that $\operatorname{deg}(B)=\operatorname{deg}\left(B_{1}\right)-1$, gen $(B)=\operatorname{gen}\left(B_{1}\right)$ and $a(B) \geq a\left(B_{1}\right)$. We have $B_{1}=\operatorname{next}(B)$ by 1$)$, so that $a(B)=a\left(B_{1}\right)=a(\operatorname{next}(B))$ and $b\left(B_{1}\right)$ $>a\left(B_{1}\right)-2$ by the definition of $\operatorname{next}(B)$.

Proposition (1.9). 1) For each pair of integers $(d, g) \quad(g \geq 0$, $\left.d \geq \operatorname{deg}\left(B_{\langle g\rangle}\right)\right)$ there exists a unique neat basic sequence with degree $d$ and genus $g$.

2) $A$ neat basic sequence $B$ with nonnegative genus $g$ satisfies the inequality $\operatorname{deg}(B) \geq \operatorname{deg}\left(B_{\langle\xi\rangle}\right)$ except in the case where $g=0$ and $\operatorname{deg}(B) \leq 2$.

Proof. 1) Apply next ( ) successively, starting with $B_{\langle g\rangle}$, and use 1) of (1.8).

2) Let $g$ be a nonnegative integer which has the expression (1.6) and $B$ a neat basic sequence such that $\operatorname{gen}(B)=g$. Suppose $a(B)<a$. If $a \geq 3$, we must have by (1.5) and 2) of (1.2) that $\operatorname{gen}(B)<g$, 
while if $a=2$, then $a(B)=1$ and the condition $0 \leq g=\operatorname{gen}(B)$ implies that $g=n=0, m=2$ and $\operatorname{deg}(B) \leq 2$. Therefore $a(B) \geq a$, in the case $g>0$ or $\operatorname{deg}(B) \geq 3$. If $\operatorname{deg}(B)<\operatorname{deg}\left(B_{\langle g\rangle}\right)$ in this case, then $B_{\langle g\rangle}=\operatorname{next}\left(B^{\prime}\right)$ for some neat basic sequence $B^{\prime}$ satisfying $a \leq a\left(B^{\prime}\right) \leq a\left(B_{\langle g\rangle}\right) \leq a+1$. Since $0 \leq b\left(B_{\langle g\rangle}\right) \leq a-2$, we find by (1.8.2) that $a\left(B^{\prime}\right) \neq a\left(B_{\langle g\rangle}\right)$, namely $a\left(B^{\prime}\right)=a$ and $a\left(B_{\langle g\rangle}\right)=a+1$. But this leads to a contradiction in the following way. We may write $B^{\prime}=\left(a ; a^{m^{\prime}},(a+1)^{a-m^{\prime}} ;(a+1)^{b^{\prime}}\right)$ with $0 \leq m^{\prime}<a, b^{\prime} \geq 0$. Since $g=\operatorname{gen}\left(B^{\prime}\right)=\operatorname{gen}\left(a ; a^{m^{\prime}},(a+1)^{a-m^{\prime}}\right)-a b^{\prime}$ and $g$ has the expression (1.6), we find $m \geq m^{\prime}$ and $(a-1)\left(m-m^{\prime}\right)-a b^{\prime}=n$ by (1.5). This implies $a\left(m-m^{\prime}-b^{\prime}\right)=\left(m-m^{\prime}\right)+n$ with $0 \leq\left(m-m^{\prime}\right)+$ $n<2 a$, therefore $m=m^{\prime}, n=b^{\prime}=0$ or $m-m^{\prime}-b=1, a=m+n-m^{\prime} \leq m+n$ 。 Hence $a\left(B_{\langle g\rangle}\right)$ must coincide with $a$ by the definition of $B_{\langle g\rangle}$. We thus have $\operatorname{deg}(B) \geq \operatorname{deg}\left(B_{\langle g\rangle}\right)$ except in the case where $g=0$ and $\operatorname{deg}(B) \leq 2$.

Remark (1.10). With the use of (1.4) and (1.7), one can prove the inequality

$$
\frac{3 a(a+1)}{(a-1)(a-2)(2 a+3)} g+1 \geq \operatorname{deg}\left(B_{\langle g\rangle}\right)
$$

for $g=\operatorname{gen}\left(a ; a^{m},(a+1)^{a-m}\right)+n, a \geq 3,0 \leq m \leq a, 0 \leq n<a-1$. The coefficient of $g$ is a strictly decreasing function of $a(a \geq 3)$ and takes the values $2,10 / 11,15 / 26, \cdots$ for $a=3,4,5, \cdots$. Therefore, if $g \geq 11$ (or $a \geq 4$ ), we have $g+3 \geq \operatorname{deg}\left(B_{\langle g\rangle}\right)$. This relation holds also for smaller values of $g$ as seen by the following table of $\operatorname{deg}\left(B_{\langle g\rangle}\right)$.

\begin{tabular}{|c|c|c|c|c|c|c|c|c|c|c|c|}
\hline$a$ & \multicolumn{2}{|c|}{2} & \multicolumn{4}{|c|}{3} & \multicolumn{3}{|c|}{4} \\
\hline$g$ & 0 & 1 & 2 & 3 & 4,5 & 6,7 & 8,9 & 10 & 11 & $12,13,14$ & $15,16,17$ \\
\hline $\operatorname{deg}\left(B_{\langle g\rangle}\right)$ & 3 & 4 & 5 & 6 & 7 & 8 & 9 & 10 & 11 & 12 \\
\hline
\end{tabular}

Now we pass on to a description of known examples of smooth irreducible curves with neat basic sequences. Recall that a curve $X$ in $\mathbb{P}^{3}$ is said to be of maximal rank if it satisfies $h^{0}\left(\mathscr{I}_{X}(n)\right) \times$ $h^{1}\left(\mathscr{I}_{X}(n)\right)=0$ for all integers $n$ (see [5]).

Lemma (1.11). A curve $X$ in $\mathbb{P}^{3}$ has maximal rank if and only if $b=0$ or $b \geq 1, a \leq n_{a+j} \leq a+1$ for all $j(1 \leq j \leq b)$, where $a=a(B(X))$, 
$b=b(B(X))$ and $n_{a+1}, \ldots, n_{a+b}$ denotes the tail of $B(X)$.

Proof. If $b=0, X$ is projectively Cohen-Macaulay and $h^{1}\left(\mathscr{I}_{X}(n)\right)$ $=0$ for all $n$ (cf. [2;Remark 1.5] and [9]). Suppose $b \geq 1$. Since $M^{\prime}(X)$ has the minimal free resolution

$$
\bigoplus_{i=1}^{r_{1}} S\left[\varepsilon_{i}^{1}-2\right] \longrightarrow \bigoplus_{j=1}^{b} S\left[n_{a+j}-2\right] \longrightarrow M^{\prime}(X) \longrightarrow 0
$$

as an $S$-module induced from $(*)$ by duality (see Introduction), we have

$$
\max \left\{n \mid M(X)_{n} \neq 0\right\}=n_{a+b}-2
$$

and $X$ is of maximal rank if and only if $n_{a+b}-2 \leq a-1$, that is if and only if $a \leq n_{a+j} \leq a+1 \quad(1 \leq j \leq b)$ by (I. 1$)$.

Thus a curve with neat basic sequence is automatically a curve of maximal rank. Let $d, g$ be integers which satisfy $d \geq \operatorname{deg}\left(B_{\langle g\rangle}\right)$, $g \geq 0$ and let $B$ be the unique neat basic sequence such that $\operatorname{deg}(B)=d$, $\operatorname{gen}(B)=g$. Put $a=a(B), b=b(B)$.

Example (1.12). Suppose $d \geq g+3, g \geq 0$ (see (1.10)). By [5], there is a smooth irreducible curve $X$ of degree $d$ and genus $g$ having maximal rank such that $h^{1}\left(\mathcal{O}_{X}(1)\right)=0$. Let $K_{B}(x)$ and $K_{B(X)}(x)$ be the polynomials defined as in the proof of (1.8). We have $K_{B}(n)=K_{B(X)}(n)=\left(\begin{array}{c}n+3 \\ 3\end{array}\right)-h^{0}\left(\mathcal{O}_{X}(n)\right)$ for $n \geq 1$. Since $a-1=\max$ $\left\{n \mid K_{B}(n) \leq 0, n \in \mathbb{Z}\right\}$ and $X$ is of maximal rank, we see $a(B(X))=a$ 。

1) If all the entries of $B$ coincide with $a$, then $h^{1}\left(\mathscr{I}_{X}(a-2)\right)=$ $-K_{B}(a-2)=b, h^{1}\left(\mathscr{I}_{X}(n)\right)=0$ for $n \geq a-1$ and $h^{0}\left(\mathscr{I}_{X}(a)\right)=K_{B}(a)=$ $a+b+1$. This implies first that the tail of $B(X)$ is $a^{b}$ by (1.11.1) and then that $B(X)=B$. It follows from the inequalities $d \geq g+3, g \geq 0$ and the formula (1.1) that

$$
\frac{1}{3}(a-3)(a+2) \leq b \leq \frac{1}{6}(a-2)(2 a+3) .
$$

2) If all the entries of the body and the tail of $B$ coincide with $a+1$, then $h^{1}\left(\mathscr{I}_{X}(a-1)\right)=-K_{B}(a-1)=b, h^{1}\left(\mathscr{I}_{X}(n)\right)=0$ for $n \geq a$, $h^{0}\left(\mathscr{I}_{X}(a)\right)=K_{B}(a)=1$ and $h^{0}\left(\mathscr{I}_{X}(a+1)\right)=K_{B}(a+1)=a+b+4$. In this case the entries of the tail of $B(X)$ must be $a+1$, therefore $B(X)=B$. The inequalities $d \geq g+3, g \geq 0$ imply 


$$
\frac{(a-2)\left(a^{2}+2 a-6\right)}{3(a-1)} \leq b \leq \frac{(a-1)\left(2 a^{2}+5 a-6\right)}{6 a} .
$$

If $K_{B}(a)=2$, we find similarly that $B(X)=B$.

3 ) In the remaining cases, the size of the tail of $B(X)$ cannot be determined in an obvious manner. So far, we can only say that $B(X)=\left(a ; a^{m^{\prime}},(a+1)^{a-m^{\prime}} ; a^{n^{\prime}},(a+1)^{b^{\prime}-n^{\prime}}\right)$ with $m^{\prime}+n^{\prime}+1=K_{B}(a), b^{\prime} \geq b$ and $b^{\prime}-n^{\prime}=h^{1}\left(\mathscr{I}_{X}(a-1)\right)$, though it is natural to expect that $B(X)=B$. For a generic $X$, this is perhaps true.

Example (1.13). If $B$ satisfies one of the following conditions, it is realised by smooth irreducible curves.

1) $b=0 \quad[7 ;$ Théorèm 2.5],

2) $b=1[1 ;$ Proposition 4.4] (cf. Appendix),

3) $B=\left(a ; a^{a} ; a^{b}\right) \quad(0 \leq 2 b \leq a)$ or $B=\left(a ; a^{m},(a+1)^{a-m} ;(a+1)^{b}\right) \quad(0 \leq$ $2 b \leq a-m)$ [2; Theorem 4.4], [3; Corollary. 2.6], [7; pp. 125-126] (cf. Appendix).

Remark (1.14). 1) In 3) of (1.12) we can show in fact that $m^{\prime} \geq 2$ if $n^{\prime}>0$, which means that $B(X)$ is neat at least in the case $K_{B}(a)=3$. The proof of this, however, requires subtle consideration on the relation matrices $\lambda_{2}, \lambda_{3}$ (cf. $[2 ; \S 1]$ and $[3 ; \S 1]$ ), so we omit the detail.

2) When $d$ is small as compared with $g$, it may occur that there are many kinds of curves of maximal rank with the same degree $d$ and genus $g$. For instance, set

$$
B_{q, r}^{\prime}=\left(a ; a^{p-r},(a+1)^{a-p-q+r},(a+2)^{q} ; a^{r},(a+1)^{q}\right)
$$

for $0 \leq r \leq(p+1) / 6,0 \leq q \leq \min (a-p+r,(a-2 p+4 r) / 6)$ and

$$
B_{q, r}^{\prime \prime}=\left(a ; a^{p-r-1},(a+1)^{a-p-q+r-1},(a+2)^{q+1}, a+3 ; a^{r},(a+1)^{q+4}\right)
$$

for $0 \leq r \leq p / 6,0 \leq q \leq \min (a-p+r-1,(a-2 p+4 r-20) / 6)$, where $a$ and $p$ are sufficiently large fixed integers. They are all realized by smooth, irreducible and arithmetically Buchsbaum curves with

$$
d=a(a+1) / 2+a-p, g=(a-1)(a-2)(2 a+3) / 6+(a-1)(a-p),
$$

which have maximal rank by (1.11) (for the proof see [4]). The basic sequences $B_{0,0}^{\prime}$ and $B_{0,0}^{\prime \prime}$ represent distinct irreducible components $\overline{\mathrm{H}}\left(B_{0,0}^{\prime}\right)$ and $\overline{\mathrm{H}}_{a B}\left(B_{0,0}^{\prime \prime}\right)$ of $\mathrm{Hilb}\left(\mathbb{P}^{3}\right)$ (see $[3 ; \mathrm{pp} .776,778]$ for notation) and probably curves with $B_{q, r}^{\prime}\left(\right.$ resp. $\left.B_{q, r}^{\prime \prime}\right)$ belong to $\overline{\mathbb{H}}\left(B_{0,0}^{\prime}\right)$ (resp. 
$\overline{\mathrm{H}}_{a . B .}\left(B_{0,0}^{\prime \prime}\right)$ ) (cf. $[2 ; \S 5]$ ). There may be other possibilities of curves of maximal rank with the above $(d, g)$, but here we won't go further in this direction.

\section{§2. Preliminary Results}

Let $\left(\alpha_{i}\right)_{i \geq 1}$ and $\left(\beta_{i}\right)_{i \geq 1}$ be sequences of elements of $k \backslash\{0\}$ such that $\alpha_{i} \neq \alpha_{j}, \beta_{i} \neq \beta_{j}$ for $i \neq j$ and set

$$
y_{i}=x_{1}-\alpha_{i} x_{3}, \quad z_{i}=x_{2}-\beta_{i} x_{4}
$$

for all $i \geq 1$. Denote by $P_{i}^{1}\left(\right.$ resp. $P_{j}^{2}$ ) the point of $\mathbf{P}^{3}$ defined by $y_{i}=x_{2}=x_{4}=0 \quad$ (resp. $\left.z_{j}=x_{1}=x_{3}=0\right)$ and by $L_{i, j}$ the line passing through $P_{i}^{1}$ and $P_{j}^{2}$, i. e. $L_{i, j}=\operatorname{Proj} R /\left(\nu_{i}, z_{j}\right)$. Let $a$ be a positive integer. We associate with a finite subset $A$ of the double indexing set $\{(i, j) \mid 1 \leq i \leq a, 1 \leq j \leq a\}$ the curve $\underset{(i, j) \in A}{\cup} L_{i, j}$ with reduced structure, which we will denote by $Z(A)$. There is a neighborhood of $Z(A)$ in $\mathbf{P}^{3}$ covered by the open sets $U_{i}^{1}(1 \leq i \leq a)$ and $U_{j}^{2}(1 \leq j \leq a)$ which are defined to be the complements of the divisors

$$
x_{1}\left(\prod_{l=1}^{a} y_{l}\right) / y_{i}=0 \quad(1 \leq i \leq a) \quad \text { and } \quad x_{2}\left(\prod_{l=1}^{a} z_{l}\right) / z_{j}=0 \quad(1 \leq j \leq a)
$$

respectively. Put $A_{\imath}^{1}=\{l \mid(i, l) \in A\}, A_{i}^{2}=\{l \mid(l, i) \in A\}$ and let $\left|A_{i}^{l}\right|$ $(l=1,2)$ denote the number of the elements. We set

$$
F_{i}^{1}=y_{i} / x_{1}, F_{i}^{2}=z_{i} / x_{2}
$$

and

$$
G_{i}^{1}=\left(\prod_{l \in A_{i}^{1}} z_{l}\right) / x_{1}^{\left|A_{i}^{1}\right|}, G_{i}^{2}=\left(\prod_{l \in A_{i}^{2}} y_{l}\right) / x_{2}^{\left|A_{i}^{2}\right|}
$$

for $1 \leq i \leq a$,

where $G_{i}^{l}=1$ if $A_{i}^{l}=\phi(l=1,2)$. Then

$$
\left.\mathscr{I}_{Z(A)}\right|_{U_{i}^{l}}=\left(F_{i}^{l}, G_{i}^{l}\right) \mathcal{O}_{U_{i}^{l}}(l=1,2,1 \leq i \leq a) .
$$

Since $Z(A)$ is a local complete intersection, its normal sheaf

$$
N_{Z(A)}:=\mathscr{H}_{\operatorname{tom}_{O_{Z(A)}}}\left(\mathscr{I}_{Z(A)} / \mathscr{I}_{Z(A)}^{2}, \mathcal{O}_{Z(A)}\right)
$$

is locally free with local trivializations

$$
\left.N_{Z(A)}\right|_{U_{i}^{l} \cap Z(A)} \cong \mathcal{O}_{U_{i}^{l} \cap Z(A)} \cdot\left(F_{i}^{l}\right) * \oplus \mathcal{O}_{U_{i}^{l} \cap Z(A)} \cdot\left(G_{i}^{l}\right) *
$$

$(l=1,2,1 \leq i \leq a)$, where $\left(F_{i}^{l}\right)^{*},\left(G_{i}^{l}\right)^{*}$ denotes the dual basis associated naturally with $F_{i}^{l}, G_{i}^{l}$. 
Proposition (2.3)。 $N_{Z(A)}$ is the direct sum of the line subbundles $Q_{Z(A)}^{1}$ and $Q_{Z(A)}^{2}$ defined by the conditions

$$
\begin{aligned}
& \begin{cases}\left.Q_{Z(A)}^{1}\right|_{U_{\imath}^{1} \cap Z(A)} \cong \mathcal{O}_{U_{i}^{1} \cap Z(A)} \cdot\left(G_{i}^{1}\right)^{*} & (1 \leq i \leq a) \\
\left.Q_{Z(A)}^{1}\right|_{U_{j}^{2} \cap Z(A)} \cong \mathcal{O}_{U_{j}^{2} \cap Z(A)} \cdot\left(F_{j}^{2}\right)^{*} & (1 \leq j \leq a)\end{cases} \\
& \begin{cases}\left.Q_{Z(A)}^{2}\right|_{U_{i}^{1} \cap Z(A)} \cong \mathcal{O}_{U_{\imath}^{1} \cap Z(A)} \cdot\left(F_{\imath}^{1}\right)^{*} & (1 \leq i \leq a) \\
\left.Q_{Z(A)}^{2}\right|_{U_{j}^{2} \cap Z(A)} \cong \mathcal{O}_{U_{j}^{2} \cap Z(A)} \cdot\left(G_{j}^{2}\right)^{*} & (1 \leq j \leq a) .\end{cases}
\end{aligned}
$$

Proof. We have $U_{\imath}^{1} \cap U_{j}^{1} \cap Z(A)=\phi, U_{\imath}^{2} \cap U_{j}^{2} \cap Z(A)=\phi$ for $i \neq j$ and $U_{i}^{1} \cap U_{j}^{2} \cap Z(A) \neq \phi$ if and only if $(i, j) \in A$, in particular no three distinct $U_{\imath}^{l} \cap Z(A)$ 's have nonempty intersection. Furthermore, if $(i, j) \in A$, then $U_{i}^{1} \cap U_{j}^{2} \cap Z(A)=L_{i, j} \backslash\left\{P_{i}^{1}, P_{j}^{2}\right\}, j \in A_{i}^{1}$ and $i \in A_{\jmath}^{2}$, therefore both

$$
F_{\imath}^{1} / G_{j}^{2}=\left(y_{i} x_{2}^{\left|A_{j}^{2}\right|}\right) /\left(x_{1} \prod_{l \in A_{j}^{2}} y_{l}\right) \quad \text { and } \quad F_{j}^{2} / G_{\imath}^{1}=\left(z_{j} x_{1}^{\left|A_{\imath}^{1}\right|}\right) /\left(x_{2} \prod_{l \in A_{\imath}^{1}} z_{l}\right)
$$

vanish nowhere on $U_{i}^{1} \cap U_{3}^{2} \cap Z(A)$. It follows from this that $\left(G_{\imath}^{1}\right)^{*}$ and $\left(F_{j}^{2}\right) *$ (resp. $\left(F_{i}^{1}\right)^{*}$ and $\left.\left(G_{j}^{2}\right)^{*}\right)(1 \leq i \leq a, 1 \leq j \leq a)$ can be patched together to form a line subbundle $Q_{Z(A)}^{\frac{1}{Z}}\left(\operatorname{resp} . Q_{Z(A)}^{2}\right)$ of $N_{Z(A)}$ and we have $N_{Z(A)}=Q_{Z(A)}^{1} \oplus Q_{Z(A)}^{2}$.

Let $A^{\prime}$ be a subset of $A$,

$$
\begin{aligned}
& A^{\prime \prime}=\left\{l \mid(i, l) \in A^{\prime}\right\}, A^{\prime 2}=\left\{l \mid(l, i) \in A^{\prime}\right\},
\end{aligned}
$$

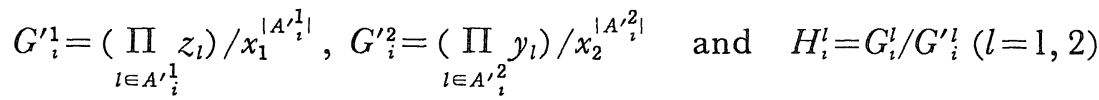

for $1 \leq i \leq a$. The line bundles $Q_{Z\left(A^{\prime}\right)}^{1}$ and $Q_{Z\left(A^{\prime}\right)}^{2}$ are connected to $Q_{Z(A)}^{1}$ and $Q_{Z(A)}^{2}$ by the following exact sequences (cf. $[12 ;(5.1)]$ );

$$
\begin{aligned}
& \left.0 \longrightarrow Q_{Z(A)}^{l} \otimes \mathscr{I}_{Z\left(A^{\prime}\right) / Z(A)} \longrightarrow Q_{Z(A)}^{l} \longrightarrow Q_{Z(A)}^{l}\right|_{Z\left(A^{\prime}\right)} \longrightarrow 0, \\
& \left.0 \longrightarrow Q_{Z\left(A^{\prime}\right)}^{l} \stackrel{\psi^{l}}{\longrightarrow} Q_{Z(A)}^{l}\right|_{Z\left(A^{\prime}\right)} \stackrel{\omega^{l}}{\longrightarrow} E_{Z\left(A^{\prime}\right) / Z(A)}^{l} \longrightarrow 0,
\end{aligned}
$$

where

$$
E_{Z\left(A^{\prime}\right) / Z(A)}^{l}=\bigoplus_{i=1}^{a} E_{Z\left(A^{\prime}\right) / Z(A), i}^{l}, E_{Z\left(A^{\prime}\right) / Z(A), i}^{l}=\iota_{P_{i}^{l} *}\left(k\left[U_{i}^{l}\right] /\left(F_{i}^{l}, G_{i}^{\prime l}, H_{i}^{l}\right)\right),
$$

$\iota_{P_{i}^{l}}: P_{i}^{l} \longrightarrow Z\left(A^{\prime}\right)$ the inclusions $(1 \leq i \leq a)$, and $\psi^{l}$ is induced by 
the natural map $\mathscr{I}_{Z(A)} / \mathscr{I}_{Z(A)}^{2} \rightarrow \mathscr{I}_{Z\left(A^{\prime}\right)} / \mathscr{I}_{Z\left(A^{\prime}\right)}^{2}(l=1,2)$. Note that $\psi^{l}$ is an isomorphism on $Z\left(A^{\prime}\right) \backslash\left\{P_{i}^{l} \mid 1 \leq i \leq a\right\}$ and that it coincides with the multiplication by $H_{i}^{l}$ at $P_{i}^{l}$. The following is an easy

Lemma (2.6). Suppose that $\mathrm{H}^{1}\left(Q_{Z(A)}^{l}\right)=0$ and that

$$
\mathrm{H}^{0}\left(\left.Q_{Z(A)}^{l}\right|_{Z\left(A^{\prime}\right)}\right) \stackrel{\omega^{l}}{\longrightarrow} \mathrm{H}^{0}\left(E_{Z\left(A^{\prime}\right) / Z(A)}^{l}\right)
$$

is surjective. Then $\mathrm{H}^{1}\left(Q_{Z\left(A^{\prime}\right)}^{l}\right)=0$.

We will need the fundamental result on the smoothability of singular curves observed by Hartshorne-Hirschowitz [10] and Sernesi [12], under a slightly weaker condition. Let $X$ be a reduced curve in $\mathbf{P}^{3}$ whose singular points are all plane singularities. Let $N_{X}$ denote the normal sheaf of $X$ and $T_{X}^{1}$ the $T^{1}$-functor as in $[10 ;$ p. 100].

Proposition (2.7). ([10; Proposition 1. 1] and [12; (1.6) Proposition]). Suppose that $\mathrm{H}^{1}\left(N_{X}\right)=0$ and that for each singular point $P$ of $X$, the natural map $\mathrm{H}^{0}\left(N_{X}\right) \rightarrow \mathrm{H}^{0}\left(\left.T_{X}^{1}\right|_{P}\right)$ is surjective. Then $X$ is flatly smoothable in $\mathbf{P}^{3}$.

Proof. Since the stalk of $T_{X}^{1}$ at $P$ is the local deformation space of a plane singularity, a nonzero element of $\left.T_{X}^{1}\right|_{P}$ corresponds to a deformation which strongly smooths $P$. Thus the proof of [10] works well for our case, too.

Corollary (2.8). Suppose that $\mathrm{H}^{1}\left(Q_{z\left(A^{\prime}\right)}^{l}\right)=0 \quad(l=1,2)$ and that for each point $P_{i}^{l}(l=1,2,1 \leq i \leq a)$, the natural map $\mathrm{H}^{0}\left(Q_{Z\left(A^{\prime}\right)}^{l}\right) \rightarrow \mathrm{H}^{0}\left(\left.Q_{Z\left(A^{\prime}\right)}^{l}\right|_{P_{i}^{l}}\right)$ is surjective. Then $Z\left(A^{\prime}\right)$ is flatly smoothable in $\mathbf{P}^{3}$.

Proof. Clear by $\left.\left.T_{Z\left(A^{\prime}\right)}^{1}\right|_{P_{i}^{l}} \cong Q_{Z\left(A^{\prime}\right)}^{l}\right|_{P_{i}^{l}}$ and $N_{Z\left(A^{\prime}\right)}=Q_{Z\left(A^{\prime}\right)}^{1} \oplus Q_{Z\left(A^{\prime}\right)}^{2}$.

Concerning the deformation of curves with neat basic sequences, we have the following

Lemma (2.9)。 Let $\pi: \mathscr{X} \rightarrow T$ be a flat family of curves in $\mathbf{P}^{3}$ and suppose that $X:=\mathscr{X}_{0}(o \in T)$ has a neat basic sequence. Then $B\left(\mathscr{X}_{v}\right)=B(X)$ for all $v$ in a neighborhood of $o$, where $\mathscr{X}_{v}=\pi^{-1}(v)(v \in T)$. 
Proof. Since $X$ is of maximal rank, there is by upper semicontinuity a neighborhood $V^{\prime}$ of $o$ such that $I_{\mathscr{X}, n}:=\left.\pi_{*}\left(\mathscr{I}_{\mathscr{X}}(n)\right)\right|_{V^{\prime}}$ are locally free with

$$
\begin{aligned}
& I_{\mathscr{X}, n} \otimes k(v) \cong \mathrm{H}^{0}\left(\mathscr{I}_{\mathscr{X}_{v}}(n)\right), \\
& h^{0}\left(\mathscr{I}_{\mathscr{X}_{v}}(n)\right)=h^{0}\left(\mathscr{I}_{X}(n)\right)
\end{aligned}
$$

for all $v \in V^{\prime}, n \geq 0$. Let $\left(a ; a^{m},(a+1)^{a-m}\right)(0 \leq m \leq a)$ be the principal part of $B(X)$ and $L$ a generic line in $\mathbb{P}^{3}$ not intersecting $X$. For each $n \geq 0$, it follows from (2.9.1) that

$$
\delta(v, n):=\operatorname{dim}_{k} \operatorname{Im}\left(\mathrm{H}^{0}\left(\mathscr{I}_{\mathscr{X}_{v}}(n)\right) \longrightarrow \mathrm{H}^{0}\left(\left.\mathscr{I}_{\mathscr{X}_{v}}(n)\right|_{L}\right)\right)
$$

is a lower semicontinuous function of $v \in V^{\prime}$ which satisfies

$$
\delta(o, n)=\left(\begin{array}{c}
n-a+1 \\
1
\end{array}\right)_{+}+m\left(\begin{array}{c}
n-a \\
0
\end{array}\right)_{+}+(a-m)\left(\begin{array}{c}
n-a-1 \\
0
\end{array}\right)_{+}
$$

by (I. 3), so $\delta(v, a) \geq m+1, \delta(v, a+1) \geq a+2$ for all $v$ lying in a neighborhood $V \subset V^{\prime}$ of $o$. Since $h^{0}\left(\mathscr{I}_{\mathscr{X}_{v}}(a-1)\right)=0$ and $h^{0}\left(\mathscr{I}_{\mathscr{X}_{v}}(a)\right)$ $=m+1$ by $(2.9 .2)$, we have $\delta(v, a-1)=0, \delta(v, a)=m+1, \delta(v, a+1)=$ $a+2$ and the principal part of $B\left(\mathscr{X}_{v}\right)$ coincides with $\left(a ; a^{m},(a+1)^{a-m}\right)$. Hence $B\left(\mathscr{X}_{v}\right)=B(X)$ for all $v \in V$ by (2.9.2) and (I. 2).

In the proof of Theorem (I. 4), the hypotheses of (2.6) and (2.8) will be verified with the use of the lemmas below.

Let $\left(\gamma_{i}\right)_{i \geq 1}$ be a sequence of elements of $k$ such that $\gamma_{i} \neq \gamma_{j}$ for $i \neq j, s$ and $t$ indeterminates over $k$ and $u_{i}=s-\gamma_{i} t(i \geq 1)$. Given integers $n, r$ satisfying $0 \leq r \leq n$, we set

$$
g_{r}^{(n)}=\left(\prod_{l=1}^{r} u_{l}\right)\left(\prod_{l=1}^{n-r} u_{2 l+r}\right),
$$

where $\prod_{l=p}^{q} u_{l}$ is understood to be 1 if $p>q$.

Lemma (2.11). 1) Fix $n(n \geq 1)$. Then the polynomials $g_{r}^{(n-1)} u_{2 n-r-1}$ $(0 \leq r \leq n-2), g_{n-1}^{(n-1)} s$ and $g_{n-1}^{(n-1)} t$ are linearly independent over $k$.

2) Fix $n(n \geq 0)$. Then the polynomials $g_{r}^{(n)}(0 \leq r \leq n)$ are linearly independent over $k$.

Proof. 1) The case $n=1$ is trivial. Suppose that $n>1$ and that

$$
\sum_{r=0}^{n-2} c_{r} g_{r}^{(n)} u_{2 n-r-1}+c_{n-1} g_{n-1}^{(n-1)} s+c_{n} g_{n-1}^{(n-1)} t=0
$$


with $c_{r} \in k(0 \leq r \leq n)$. Then since $u_{1}$ divides all $g_{r}^{(n-1)}(1 \leq r \leq n-1)$ but not $g_{0}^{(n-1)}$, we have $c_{0}=0$. Dividing the above relation by $u_{1}$, we find that $c_{r}=0$ for all $1 \leq r \leq n$ by induction on $n$.

2) Similar to 1).

For each $\nu \geq 0$ we denote by $k[s, t]_{\nu}$ the homogeneous part of $k[s, t]$ of degree $\nu$.

Corollary (2.12). Given integers $m, n$ satisfying $n \geq 1,0 \leq m \leq n$, let $V_{m, n}\left(\right.$ resp. $\left.V_{m, n}^{\prime}\right)$ be the linear subspace of $k[s, t]$ spanned over $k$ by $\left\{g_{r}^{(n-1)} u_{2 n-r-1} \mid m \leq r \leq n-2\right\} \cup\left\{g_{n-1}^{(n-1)} s, g_{n-1}^{(n-1)} t\right\} \quad\left(\right.$ resp. $\left.\left\{g_{r}^{(n)} \mid m \leq r \leq n\right\}\right)$. Then $V_{m, n}=V_{m, n}^{\prime}=g_{m}^{(m)} k[s, t]_{n-m}(0 \leq m<n)$ and $V_{n, n} \supset V_{n, n}^{\prime}=g_{n}^{(n)} k(n \geq 1)$.

Proof. The inclusion $V_{n, n} \supset g_{n}^{(n)} k(n \geq 1)$ is obvious. If $0 \leq m<n$, the polynomial $g_{m}^{(m)}$ divides $g_{r}^{(n-1)}$ for all $m \leq r \leq n-1$. Since $n-m+1$ homogeneous polynomials $g_{r}^{(n-1)} u_{2 n-r-1} / g_{m}^{(m)}(m \leq r \leq n-2), g_{n-1}^{(n-1)} s / g^{(m)}$ and $g_{n-1}^{(n-1)} t / g_{m}^{(m)}$ of degree $n-m$ in $s, t$ are linearly independent over $k$ by (2.11), we have $V_{m, n}=g_{m}^{(m)} k[s, t]_{n-m}$. In the same way we get the assertion for $V_{m, n}^{\prime}(n \geq 1,0 \leq m \leq n)$.

For each $p(0 \leq p \leq a)$, let $V_{p}$ (resp. $\left.V_{p}^{\prime}\right)$ denote the linear subspace of $k[s, t]$ spanned over $k$ by $\left\{g_{r}^{(n-1)} u_{2 n-r-1} \mid r \geq 0,2 n-r-1<a\right.$, $0 \leq n-r-1 \leq p\} \cup \bigcup_{n=1}^{a}\left\{g_{n-1}^{(n-1) \cdot}(k+s k+t k)\right\} \quad$ (resp. $\left\{g_{r}^{(n)} \mid r \geq 0,2 n-r<a, 0 \leq\right.$ $n-r \leq p\})$ and put $V_{p}^{\prime \prime}=V_{p}^{\prime}+s V_{p}^{\prime}+t V_{p}^{\prime}$.

Corollary (2.13). 1) If $0 \leq \nu \leq p+1, m \geq 0, m+2 \nu \leq a$, then $g_{m}^{(m)} k[s, t]_{\nu}$ $\subset V_{p}$.

2) If $0 \leq \nu \leq p, m \geq 0, m+2 \nu<a$, then $g_{m}^{(m)} k[s, t]_{\nu} \subset V_{p}^{\prime}$.

Proof. 1) Put $n=m+\nu$. The case $n=0$ is trivial. If $n>0$, then $g_{n-1}^{(n-1)} s, g_{n-1}^{(n-1)} t$ and $g_{r}^{(n-1)} u_{2 n-r-1}(m \leq r \leq n-2)$ are all contained in $V_{p}$ by definition. Hence the assertion follows from (2.12).

2) Similar to 1).

Let $m$ and $n$ be positive integers and for each $\mu(0 \leq \mu \leq n)$ let $h_{\mu}^{\prime}$ denote a homogeneous polynomial in $s, t$ of degree $\mu$ which is not divisible by any $u_{l}(1 \leq l \leq m)$. We set $h_{\lambda}=\prod_{l=1}^{\lambda} u_{l}(0 \leq \lambda \leq m)$ and consider the ring $E:=k[s, t] /\left(h_{m}, h_{n}^{\prime}\right)$ as a $k$-vector space $(k \subset k[s, t])$. 
Lemma (2.14)。The polynomials $h_{\lambda} h_{\mu}^{\prime}(0 \leq \lambda \leq m-1,0 \leq \mu \leq n-1)$ $\left(\bmod \left(h_{m}, h_{n}^{\prime}\right)\right)$ form a basis of the mn-dimensional vector space $E$.

Proof. Since $h_{m}$ and $h_{n}^{\prime}$ are relatively prime, we have $\operatorname{dim}_{k}(E)=m n$. The assertion being obviously true for $m=1$, we proceed by induction on $m$. Suppose that $m>1$ and that

$$
\sum_{\lambda=0}^{m-1} \sum_{\mu=0}^{n-1} c_{\lambda, \mu} h_{\lambda} h_{\mu}^{\prime}=h h_{m}+h^{\prime} h_{n}^{\prime}
$$

with $c_{\lambda, \mu} \in k$ and $h, h^{\prime} \in k[s, t]$. Since $h_{\lambda}(1 \leq \lambda \leq m)$ are divisible by $u_{1}$, the relation (2.14.1) consists of the following two parts;

$$
\sum_{\mu=0}^{n-1} c_{0, \mu} h_{\mu}^{\prime} \equiv g^{\prime} h_{n}^{\prime} \quad\left(\bmod u_{1}\right)
$$

(2. 14.3) $\sum_{\lambda=1}^{m-1} \sum_{\mu=0}^{n-1} c_{\lambda, \mu}\left(h_{\lambda} / u_{1}\right) h_{\mu}^{\prime}=h\left(h_{m} / u_{1}\right)+g h_{n}^{\prime}+\left(g^{\prime} h_{n}^{\prime}-\sum_{\mu=0}^{n-1} c_{0, \mu} h_{\mu}^{\prime}\right) / u_{1}$,

where $h^{\prime}=g u_{1}+g^{\prime}$ with $g^{\prime} \in k[t]$. It follows from (2.14.2) that $g^{\prime}=0$, $c_{0, \mu}=0(0 \leq \mu \leq n-1)$ for reasons of degree, and hence from (2.14.3) follows that $c_{\lambda, \mu}=0 \quad(1 \leq \lambda \leq m-1,0 \leq \mu \leq n-1)$ by induction. This proves our assertion.

Suppose $a \geq 2$. Let $\tau=\left\{i_{1}, \ldots, i_{p+1}\right\}, \quad \bar{\tau}=\left\{j_{1}, \ldots, j_{a-p-1}\right\} \quad\left(i_{1}<i_{2}<\cdots\right.$ $\left.<i_{p+1}, j_{1}<i_{2}<\cdots<j_{a-p-1}, 0 \leq p \leq a-2\right)$ be subsets of $\tau_{a}:=\{i \mid 1 \leq i \leq a\}$ such that $\bar{\tau}=\tau_{a} \backslash \tau_{\text {. We }}$ set $h_{\lambda}^{\tau}=\prod_{l=1}^{\lambda} u_{\imath l}(0 \leq \lambda \leq p+1), h_{\mu}^{*}=\prod_{l=1}^{\mu} u_{j_{l}}(0 \leq \mu \leq a-p-1)$ and $E^{\tau}=k[s, t] /\left(h_{p+1}^{\tau}, h_{a-p-1}^{\tau}\right)$. Let $\kappa^{\tau}: k[s, t] \rightarrow E^{\tau}$ denote the natural map. Later we will have to consider three cases;

case 1) $\tau=\{a-2 p+2 l-3 \mid 1 \leq l \leq p+1\}$ with $0 \leq p<[a / 2]$,

case 2) $\tau=\{a-2 \mathrm{p}\} \cup\{a-2 p+2 l-1 \mid 1 \leq l \leq p\}$ with $0 \leq p \leq[(a-1) / 2]$, case 3) $\tau=\{a-2 l \mid 1 \leq l \leq p+1\}$ with $0 \leq p<[(a-1) / 2]$.

Lemma (2.15). We have $h_{p+1}^{\tau}, h_{\lambda}^{\tau} h_{\mu}^{\tau}(0 \leq \lambda \leq p, 0 \leq \mu \leq a-p-2) \in V_{p}^{\prime \prime}$ $\left(\right.$ resp. $\left.V_{p}\right)$ and $\left.\kappa^{\tau}\right|_{V_{p}^{\prime \prime}}\left(\operatorname{resp} .\left.\kappa^{\tau}\right|_{V_{p}}\right)$ is surjective in the cases 1) and 2) (resp. in the case 3)).

Proof. Clear by (2.13) and (2.14). 


\section{§ 3. Curve with $B=\left(a ; a^{a} ; a^{b}\right)$}

We will construct in this section a smoothable and connected curve of the form $Z(A)$ for each pair $(a, b)$ satisfying $a>0,0 \leq b<[a / 2]$ $\times(a-[a / 2])$, which has $\left(a ; a^{a} ; a^{b}\right)$ as its basic sequence, where $[a / 2]$ denotes the integral part of $a / 2$. The notation of the previous section will be used without change, unless otherwise specified.

Given a positive integer $a$, let $f_{i}$ denote the homogeneous polynomial $\left(\prod_{l=1}^{i} \mathrm{z}_{l}\right)\left(\prod_{l=i+1}^{a} y_{l}\right)(0 \leq i \leq a), J$ the ideal $\left(f_{0}, f_{1}, \ldots, f_{a}\right) R$ and $X_{0}$ the curve Proj $R / J$. One sees $X_{0}=Z(A)$ with $A=\{(i, j) \mid 1 \leq j \leq i \leq a\}$. Put $\rho(a)=[a / 2](a-[a / 2]) . \quad$ An integer $b(1 \leq b \leq \rho(a))$ can be written uniquely in the form $p(a-p)+q(1 \leq q \leq a-2 p-1,0 \leq p<[a / 2])$, since $\rho(a)=\sum_{l=1}^{p}(a-2 l+1)+\sum_{l=p+1}^{[a / 2]}(a-2 l+1)$ for $0 \leq p \leq[a / 2]$. With the help of this expression, we define $A(b)$ to be the set

$$
A \backslash(\{(j+2 l-1, j) \mid 1 \leq j \leq a-2 l+1,1 \leq l \leq p\} \cup\{(j+2 p+1, j) \mid 1 \leq j \leq q\})
$$

for $1 \leq b \leq \rho(a)$ and denote $Z(A(b))$ by $X_{b}$ and $X_{p(a-p)}$ by $Y_{p}(0 \leq p \leq$ $[a / 2])$. The curve $X_{b}$ is obtained practically by removing $q$ lines $L_{j+2 p+1, j}(1 \leq j \leq q)$ from $Y_{p}$.

Lemma (3.1). We have $h^{0}\left(\mathcal{O}_{X_{b} \cap L_{j+2 p+1, j}}\right)=a$ for $1 \leq j \leq q$ and $L_{j+2 p+1, j} \cap L_{j^{\prime}+2 p+1, j^{\prime}}=\phi$ for $j \neq j^{\prime}$.

Proof. The latter is clear. To verify the first equality, consider for each $P_{i}^{1}\left(\right.$ resp. $\left.P_{\jmath}^{2}\right)$ the number of the lines passing through it and contained in $Y_{p}$, which we denote by $d_{\imath}^{1}(p)$ (resp. $\left.d_{j}^{2}(p)\right)$. One sees without difficulty that

$$
\left\{\begin{array}{l}
d_{\imath}^{1}(p)=i-\min ([i / 2], p) \\
d_{j}^{2}(p)=(a-j+1)-\min ([(a-j+1) / 2], p)
\end{array} .\right.
$$

Since $P_{\imath}^{1}$ and $P_{j}^{2}$ are all plane singularities, we get

$$
h^{0}\left(\mathcal{O}_{X_{b} \cap L_{j+2 p+1, j}}\right)=\left(d_{j+2 p+1}^{1}(p)-1\right)+\left(d_{j}^{2}(p)-1\right)=a(1 \leq j \leq q) .
$$

This lemma enables us to compute the basic sequence of $X_{b}$ easily, combined with the following 
Lemma (3.2)。([2; Corollary A.3])。 Let $X$ be a curve in $\mathbb{P}^{3}$ and $L$ a line which intersects $X$ in a set of finite points. Suppose that $B(X \cup L)=$ $\left(a ; n_{1}, \ldots, n_{a} ; n_{a+1}, \ldots, n_{a+b}\right)$ and that $h^{0}\left(\mathcal{O}_{X \cap L}\right)=n$ with $n \geq n_{a}$. Then $B(X)=\left(a ; n_{1}, \ldots, n_{a} ; n_{a+1}, \ldots, n_{a+b}, n\right)$ up to a permutation of the tail.

Proposition (3.3). $B\left(X_{b}\right)=\left(a ; a^{a} ; a^{b}\right)$ and $X_{b}$ is connected if and only if $0 \leq b<\rho(a)$.

Proof. Since $\mathscr{I}_{x_{0}}$ has the free resolution

$$
\begin{aligned}
& 0 \longrightarrow \mathcal{O}_{\mathbf{P}^{3}}(-a-1)^{a} \stackrel{\varphi}{\longrightarrow} \mathcal{O}_{\mathbf{P}^{3}}(-a)^{a+1} \stackrel{\left(f_{f} \ldots \ldots f_{a}\right)}{\longrightarrow} \mathscr{\mathscr { I }}_{X_{0}} \longrightarrow 0 \\
& \text { with } \varphi=\left(\begin{array}{cccc}
-z_{1} & & & 0 \\
y_{1} & -z_{2} & & 0 \\
& y_{2} & \ddots & -z_{a} \\
0 & & \ddots & y_{a}
\end{array}\right) \text {, }
\end{aligned}
$$

the basic sequence of $X_{0}$ is $\left(a ; a^{a}\right)$. Apply (3.2) successively, starting with $X_{0}$, and we get $B\left(X_{b}\right)=\left(a ; a^{a} ; a^{b}\right)$. For the connectedness, observe first that

$$
X_{\rho(a)}=Z\left(A_{\text {even }}\right) \cup Z\left(A_{\text {odd }}\right) \quad \text { (disjoint union), }
$$

where $A_{\text {even }}=\{(i, j) \mid$ both $i$ and $j$ are even $\} \cap A, A_{\text {odd }}=\{(i, j) \mid$ toth $i$ and $j$ are odd $\} \cap A$. The points $P_{\imath}^{1}$ and $P_{j}^{2} \quad(1 \leq i \leq a, 1 \leq j \leq a)$ with subscripts in the same congruent class modulo 2 are contained together in either $Z\left(A_{\text {even }}\right)$ or $Z\left(A_{\text {odd }}\right)$ and are connected by lines of the forms $L_{l, l}, L_{l+2, l}$. If $0 \leq b<\rho(a)$, then $X_{b}$ contains a line $L_{l, \text { s }}$ such that $i \not \equiv j$ (mod 2), which joins the two points $P_{\imath}^{1}$ and $P_{,}^{2}$ not lying in a single connected component of $X_{\rho(a)}$, therefore $X_{b}$ is connected.

Proposition (3.4)。For each $m(1 \leq m \leq \rho(a))$, write $m=p(a-p)+q$ $(1 \leq q \leq a-2 p-1,0 \leq p<[a / 2])$ and set

$$
f_{a+m}=\left(\prod_{l=1}^{q-1} z_{l}\right)\left(\prod_{l=1}^{p+1} y_{q+2 l-2} z_{q+2 l-1}\right)\left(\prod_{l=q+2 p+2}^{a} y_{l}\right) .
$$

Then for each $b \quad(0 \leq b \leq \rho(a))$ the ideal $I_{X_{b}}$ is generated by the homogeneous polynomials $f_{n}(0 \leq n \leq a+b)$ of degree $a$.

Proof. Since $I_{X_{0}}=J$, it suffices to show that $I_{X_{m}}=I_{X_{m-1}}+f_{a+m} R$ for all $m(1 \leq m \leq \rho(a))$. In fact, the hyperplane $f_{a+m}=0$ contains the lines 
$L_{i, j}$ whose double indices $(i, j)$ lie in $A(m)=A \backslash\{(j+2 l-1, j) \mid 1 \leq j \leq q$, $1 \leq l \leq p+1 \quad$ or $q+1 \leq j \leq a, 1 \leq l \leq \min (p,[(a-j+1) / 2])\}$, but not $L_{q+2 p+1, q}$. Since $X_{m} \cup L_{q+2 p+1, q}=X_{m-1}$, this means that $f_{a+m}$ vanishes on $X_{m}$ but not on $X_{m-1}$. We see therefore by (3.3) that $I_{X_{m}}=I_{X_{m-1}}+f_{a+m} R$.

The rest of this section is devoted to the proof for the smoothability of $X_{b}(0 \leq b \leq \rho(a))$. Let us begin by describing a method to construct global sectios of $N_{X_{b}}$ and $\left.N_{X_{0}}\right|_{X_{b}}$. First set

$$
\begin{cases}H_{i}^{1}(p)=\left(\prod_{l=1}^{p} z_{i-2 l+1}\right) / x_{1}^{p} & (0 \leq p \leq[i / 2]) \\ H_{i}^{2}(p)=\left(\prod_{l=1}^{p} y_{i+2 l-1}\right) / x_{2}^{p} & (0 \leq p \leq[(a-i+1) / 2])\end{cases}
$$

and

$$
G_{i}^{1}(p)=\left(\prod_{l=1}^{i} z_{l}\right) /\left(x_{1}^{i} H_{i}^{1}(p)\right), G_{i}^{2}(p)=\left(\prod_{l=i}^{a} y_{l}\right) /\left(x_{2}^{a-i+1} H_{\imath}^{2}(p)\right)
$$

for $1 \leq i \leq a$. By $(2.1)$

$$
\begin{aligned}
\left.\mathscr{I}_{X_{b}}\right|_{U_{i}^{1}} & =\left\{\begin{array}{ll}
\left(F_{i}^{1}, G_{i}^{1}([i / 2])\right) \mathcal{O}_{U_{i}^{1}} & (1 \leq i \leq 2 p+1) \\
\left(F_{i}^{1}, G_{i}^{1}(p+1)\right) \mathcal{O}_{U_{i}^{1}} & (2 p+2 \leq i \leq 2 p+q+1) \\
\left(F_{i}^{1}, G_{i}^{1}(p)\right) \mathcal{O}_{U_{i}^{1}} & (2 p+q+2 \leq i \leq a)
\end{array},\right. \\
\left.\mathscr{I}_{X_{b}}\right|_{U_{j}^{2}} & =\left\{\begin{array}{ll}
\left(F_{j}^{2}, G_{j}^{2}(p+1)\right) \mathcal{O}_{U_{j}^{2}} & (1 \leq j \leq q) \\
\left(F_{j}^{2}, G_{j}^{2}(p)\right) \mathcal{O}_{U_{j}^{2}} & (q+1 \leq j \leq a-2 p-1) \\
\left(F_{j}^{2}, G_{j}^{2}([(a-j+1) / 2])\right) \mathcal{O}_{U_{j}^{2}} & (a-2 p \leq j \leq a)
\end{array},\right.
\end{aligned}
$$

where $b=p(a-p)+q$ with $0 \leq p<[a / 2], 1 \leq q \leq a-2 p-1$. Note that

$$
\left\{\begin{array}{l}
G_{i}^{1}(0)=f_{i} /\left(x_{1}^{i} \times \prod_{l=i+1}^{a} y_{l}\right), G_{i}^{2}(0)=f_{i-1} /\left(x_{2}^{a-i+1} \times \prod_{l=1}^{i-1} z_{l}\right) \\
F_{i}^{1}=f_{0} /\left(x_{1} \times \prod_{l=1, l \neq l}^{a} y_{l}\right), F_{i}^{2}=f_{a} /\left(x_{2} \times \prod_{l=1, l \neq i}^{a} z_{l}\right)
\end{array}\right.
$$

for $1 \leq i \leq a$. Since $\mathscr{I}_{X_{0}}$ has the free resolution (3.3.1), the sheaf $\left.N_{X_{0}}\right|_{X_{b}} \cong \mathscr{H}_{\text {tom }}\left(\mathscr{I}_{X_{0}}, \mathcal{O}_{X_{b}}\right)$ coincides with the kernel of the map

$$
\theta(b): \mathcal{O}_{X_{b}}(a)^{a+1} \stackrel{t_{\varphi}}{\longrightarrow} \mathcal{O}_{X_{b}}(a+1)^{a},
$$

and to obtain an element of $\mathrm{H}^{0}\left(\left.N_{X_{0}}\right|_{X_{b}}\right)$, we have only to compute the kernel of the map

$$
\Theta(b):\left(R_{a} / I_{X_{b}, a}\right)^{a+1} \stackrel{t_{\varphi}}{\longrightarrow}\left(R_{a+1} / I_{X_{b}, a+1}\right)^{a} .
$$


Let $\left(F_{\imath}^{1}\right)^{*},\left(G_{\imath}^{1}(0)\right)^{*}$ denote the local basis of $N_{X_{0}}$ on $U_{\imath}^{l} \cap X_{0}$ for $1 \leq i \leq a$, $l=1,2$ (see $(2,2))$ and $E_{i}^{l}(p)$ the sheaf $\iota_{P_{l *}^{l}}\left(k\left[U_{l}^{l}\right] /\left(F_{\imath}^{l}, G_{i}^{l}(p), H_{\imath}^{l}(p)\right)\right)$ on $X_{b}(l=1,0 \leq p \leq[i / 2]$ or $l=2,0 \leq p \leq[(a-i+1) / 2])$. We have the exact sequence (2.5) with $Z(A)=X_{0}, Z\left(A^{\prime}\right)=X_{b}$ and

$$
\left\{\begin{array}{l}
E_{X_{b} / X_{0}}^{1}=\left(\bigoplus_{i=1}^{2 p+1} E_{i}^{1}([i / 2])\right) \oplus\left(\bigoplus_{i=2 p+2}^{2 p+q+1} E_{i}^{1}(p+1)\right) \oplus\left(\underset{i=2 p+q+2}{\oplus} E_{l}^{1}(p)\right) \\
E_{X_{b} / X_{0}}^{2}=\left(\bigoplus_{j=1}^{q} E_{j}^{2}(p+1)\right) \oplus\left(\bigoplus_{j=q+1}^{a-2 p-1} E_{j}^{2}(p)\right) \oplus\left(\bigoplus_{j=a-2 p}^{a} E_{j}^{2}([(a-j+1) / 2])\right)
\end{array}\right.
$$

for $1 \leq b \leq \rho(a)$. Through the identification of $\mathrm{H}^{0}\left(\left.N_{X_{0}}\right|_{X_{b}}\right)$ and $\operatorname{Ker}(\Theta(b))$ as above, a vector ${ }^{t} v={ }^{t}\left(v_{\nu}\right)_{0 \leq \nu \leq a} \in \operatorname{Ker}(\Theta(b))$ corresponds to a global section $\left(\sigma_{1}, \sigma_{2}\right)\left(\sigma_{l} \in \mathrm{H}^{0}\left(\left.Q_{X_{0}}^{l}\right|_{X_{b}}\right), l=1,2\right)$ of $\left.N_{X_{0}}\right|_{X_{b}}=\left.\left(Q_{X_{0}}^{1} \oplus Q^{2}{ }_{X_{0}}^{2}\right)\right|_{X_{b}}$ which can be written in the form

$$
\begin{cases}c_{1}\left(v_{0} / x_{1}^{a}\right)\left(F_{i}^{1}\right) *+e_{t}^{1}\left(v_{i} / x_{1}^{a}\right)\left(G_{\imath}^{1}(0)\right)^{*} & \text { on } U_{\imath}^{1} \cap X_{b} \\ c_{\imath}^{2}\left(v_{a} / x_{2}^{a}\right)\left(F_{\imath}^{2}\right) *+e_{\imath}^{2}\left(v_{i-1} / x_{2}^{a}\right)\left(G_{\imath}^{2}(0)\right)^{*} & \text { on } U_{\imath}^{2} \cap X_{b}\end{cases}
$$

with suitable $c_{i}^{l}, e_{i}^{l} \in k \backslash\{0\} \quad(l=1,2,1 \leq i \leq a)$ (cf. (3.6)). Furthermore, $\left(\sigma_{1}, \sigma_{2}\right)$ comes from a global section of $N_{X_{b}}$ by way of $\left(\psi^{1}, \psi^{2}\right)$ if and only if $\omega^{1}\left(\sigma_{1}\right)=0, \omega^{2}\left(\sigma_{2}\right)=0$.

In the argument below, we will use the following term.

Definition (3.8). Let $X$ be a subscheme of $X_{0}$. By a pre-normal of $X$ we mean a row vector $v=\left(v_{\nu}\right)_{0 \leq \nu \leq a}$ with $v_{\nu} \in R_{a}(0 \leq \nu \leq a)$ such that all the components of $v \varphi$ lie in $I_{X}$.

Note that a pre-normal of $Y_{p}(0 \leq p \leq[a / 2])$ is at the same time a pre-normal of $Y_{p^{\prime}}$ for all $p^{\prime}\left(p \leq p^{\prime} \leq[a / 2]\right)$. Set $s=x_{2} / x_{1}, t=x_{4} / x_{1}$ and $u_{i}=z_{i} / x_{1}(i \geq 1)$. Denote $I_{Y_{p^{\prime}}} \cap y_{a} R$ by $D_{l}(p) \quad(0 \leq p \leq[a / 2], l \geq 1)$ and the linear subspace $\left\{f / y_{a} x_{1}^{l-1}\left(\bmod F_{a}^{1}\right) \mid f \in D_{l}(p)\right\}$ of $k[s, t]$ by $W_{l}(p)$. We have $W_{l}(p) \subset W_{l}(p+1)$ and $\left(x_{i} / x_{1}\right) W_{l}(p) \subset W_{l+1}(p)$ for $1 \leq i \leq 4$.

Lemma (3.9). Let $p, r, n$ be integers satisfying $r \geq 0,2 n-r<a$ and $0 \leq n-r \leq p \leq[a / 2]$. Then $g_{r}^{(n)} \in W_{a}(p) \quad(c f .(2.10))$.

Proof. In the case $n=r$ the assertion is clear by $f_{n} \in D_{a}(p)(0 \leq n<a)$. If $n>r$, then since $0 \leq n-r-1 \leq p-1<[a / 2], 1 \leq r+1<a-2(n-r-1)-1$ 
and $(r+1)+2(n-r-1)+2 \leq a$, the polynomial $f_{a+(n-r-1)(a-n+r+1)+(r+1)}$ defined in (3.4) belongs to $\mathrm{D}_{a}(p)$, that is $g_{r}^{(n)} \in W_{a}(p)$.

Lemma (3.10). 1) Suppose $0 \leq p<[a / 2]$. For each $\xi \in k[s, t]$, there exists a pre-normal $v^{1}(p, \xi)=\left(v_{\nu}^{1}(p, \xi)\right)_{0 \leq \nu \leq a}$ of $Y_{p}$ such that $v_{\nu}^{1}(p, \xi)=0$ for $0 \leq \nu \leq a-1$ and $v_{a}^{1}(p, \xi) / x_{1}^{a} \equiv \xi\left(\bmod \left(F_{a}^{1}, G_{a}^{1}(p+1), H_{a}^{1}(p+1)\right)\right)$.

2) There exists, for each $p(0 \leq p<[a / 2])$, a pre-normal $w^{1}(p)=$ $\left(w_{\nu}^{1}(p)\right)_{0 \leq \nu \leq a}$ of $Y_{p}$ such that $w_{\nu}^{1}(p)=0$ for $0 \leq \nu \leq a-1$ and $w_{a}^{1}(p) / x_{1}^{a} \equiv$ $H_{a}^{1}(p+1)\left(\bmod F_{a}^{1}\right)$.

3) There exists a pre-normal $w^{1}=\left(w_{\nu}^{\prime 1}\right)_{0 \leq \nu \leq a}$ of $Y_{0}$ such that $w_{\nu}^{\prime 1}=0$ for $0 \leq \nu \leq a-1$ and $w_{a}^{\prime 1} / x_{1}^{a} \equiv 1\left(\bmod F_{a}^{1}\right)$.

Proof. 1) There is a polynomial $f(p, \xi)$ of $D_{a+1}(p)$ such that $f(p, \xi) / y_{a} x_{1}^{a} \equiv \xi\left(\bmod \left(F_{a}^{1}, G_{a}^{1}(p+1), H_{a}^{1}(p+1)\right)\right)$ by (2.15) and (3.9). Setting $v_{\nu}^{1}(p, \xi)=0 \quad(0 \leq \nu \leq a-1)$ and $v_{a}^{1}(p, \xi)=f(p, \xi) / y_{a}$, we get a desired pre-normal. 2), 3) Similar to 1).

Let

$$
\pi_{i}^{1}:\left(R_{a}\right)^{a+1} \longrightarrow k\left[x_{2} / x_{1}, x_{3} / x_{1}, x_{4} / x_{1}\right] \quad(1 \leq i \leq a)
$$

and

$$
\pi_{i}^{2}:\left(R_{a}\right)^{a+1} \longrightarrow k\left[x_{1} / x_{2}, x_{3} / x_{2}, x_{4} / x_{2}\right] \quad(1 \leq i \leq a)
$$

denote the maps defined by $\pi_{i}^{1}(v)=v_{i} / x_{1}^{a}$ and $\pi_{i}^{2}(v)=v_{i-1} / x_{2}^{a}$ respectively for $v=\left(v_{\nu}\right)_{0 \leq \nu \leq a}$.

Proposition (3.11). Let $\zeta$ be an element of $k\left[x_{2} / x_{1}, x_{3} / x_{1}, x_{4} / x_{1}\right]$.

1) There exist for each pair $(i, p)(1 \leq i \leq a, 0 \leq p<[i / 2])$, pre-normals $v$, w of $Y_{p}$ satisfying $\pi_{\nu}^{1}(v)=0, \pi_{\nu}^{1}(w)=0(1 \leq \nu \leq i-1)$ such that

$$
\left\{\begin{array}{l}
\zeta-\pi_{i}^{1}(v) \equiv 0\left(\bmod \left(F_{i}^{1}, G_{i}^{1}(p+1), H_{i}^{1}(p+1)\right)\right) \\
\pi_{i}^{1}(w) \equiv H_{i}^{1}(p+1)\left(\bmod F_{i}^{1}\right)
\end{array} .\right.
$$

2) There exist for each pair $(i, p)(1 \leq i \leq a, 0 \leq p \leq[i / 2])$ pre-normals $v^{\prime}, w^{\prime}$ of $Y_{p}$ satisfying $\pi_{\nu}^{1}\left(v^{\prime}\right)=0, \pi_{\nu}^{1}\left(w^{\prime}\right)=0(1 \leq \nu \leq i-1)$ such that

$$
\left\{\begin{array}{l}
\zeta-\pi_{i}^{1}\left(v^{\prime}\right) \equiv 0\left(\bmod \left(F_{\imath}^{1}, G_{i}^{1}(p), H_{i}^{1}(p)\right)\right) \\
\pi_{i}^{1}\left(w^{\prime}\right) \equiv H_{i}^{1}(p)\left(\bmod F_{\imath}^{1}\right)
\end{array}\right.
$$

Proof. We will prove the assertions by induction on $a$. The case $a=1$ is trivial. Assume that $a>1$ and that the assertions are true 
for $a-1$. Replacing $a$ by $a-1$ in the formulae which have been described in this section, one obtains the curves, the polynomials and the maps associated with the integer $a-1$ in the same manner. We denote them by the same symbols with attached on the heads. Notice first that $Y_{p} \subset \tilde{Y}_{p} \cup \stackrel{j}{j=1}_{a, j} L_{a n d}$ and $y_{a} I_{\dddot{Y}_{p}} \subset I_{Y_{p}}(0 \leq p \leq[(a-1) / 2])$. This allows us to construct pre-normals of $Y_{p}$ from those of $\tilde{Y}_{p}$ in a canonical way. In fact, for a given pre-normal $\tilde{\eta}=\left(\tilde{\eta}_{\nu}\right)_{0 \leq \nu \leq a-1}$ of $\tilde{Y}_{p}(0 \leq$ $p \leq[(a-1) / 2])$, we can define a pre-normal $\eta=\left(\eta_{\nu}\right)_{0 \leq \nu \leq a}$ of $Y_{p}$ which satisfies $\pi_{\nu}^{1}(\eta) \equiv \tilde{\pi}_{\nu}^{1}(\tilde{\eta})\left(\bmod F_{\nu}^{1}\right)(0 \leq \nu \leq a-1)$ up to constant multiplication, by setting $\eta_{\nu}=\tilde{\eta}_{\nu} y_{a}(0 \leq \nu \leq a-1), \eta_{a}=\tilde{\eta}_{a-1} z_{a}$. Besides, the polynomials $\tilde{F}_{i}^{1}, \tilde{G}_{i}^{1}(p)$ and $\tilde{H}_{i}^{1}(p)$ coincide with $F_{i}^{1}, G_{i}^{1}(p)$ and $H_{i}^{1}(p)$ respectively for each pair $(i, p)(1 \leq i \leq a-1,0 \leq p \leq[i / 2])$. The assertions 1$)$ and 2$)$ therefore hold for $1 \leq i \leq a-1$ by the induction hypotheses and it remains to deal with the case $i=a$. Now suppose $i=a$ and let $v^{1}(p, \xi)$ and $w^{1}(p) \quad(0 \leq p<[a / 2])$ be the pre-normals described in (3.10) with $\xi \equiv \zeta\left(\bmod F_{a}^{1}\right)$. It suffices to set $v=v^{1}(p, \xi)$ and $w=w^{1}(p)$. We have thus proved 1) completely. Since a pre-normal of $Y_{p}$ is at the same time a pre-normal of $Y_{p+1}(0 \leq p<[a / 2])$, the proof above gives also a proof of 2) for $i=a, 0<p \leq[a / 2]$. When $i=a$ and $p=0$, we use the pre-normal $w^{\prime 1}$ described in (3.10) to verify 2$)$. In this case $H_{a}^{1}(0)=1$, hence the assertion is clear.

Let $b=p(a-p)+q \quad(0 \leq p<[a / 2], 1 \leq q \leq a-2 p-1)$ be an integer such that $1 \leq b \leq \rho(a)$. The transposed vector of a pre-normal $v$ of $Y_{p}$ gives an element of $\operatorname{Ker}(\Theta(b))$ corresponding to a global section $\left(\sigma_{1}, \sigma_{2}\right)$ of $\left.N_{X_{0}}\right|_{X_{b}}=\left.\left(Q_{X_{0}}^{1} \oplus Q_{X_{0}}^{2}\right)\right|_{X_{b}}$ as explained before. Locally, $\sigma_{l} \equiv \pi_{i}^{l}(v)$ 。 $\left(G_{i}^{l}(0)\right) *\left(\bmod H^{0}\left(\mathscr{I}_{U_{l}^{l} \cap X_{b}}\right)\right)$ up to constant multiplication on $U_{i}^{l} \cap X_{b}$ $(l=1,2,1 \leq i \leq a)$ (cf. (3.7)), therefore we get the following result.

Corollary (3.12)。1) The complex

$$
0 \longrightarrow \mathrm{H}^{0}\left(Q_{X_{b}}^{l}\right) \stackrel{\psi^{l}}{\longrightarrow} \mathrm{H}^{0}\left(\left.Q_{X_{0}}^{l}\right|_{X_{b}}\right) \stackrel{\omega^{l}}{\longrightarrow} \mathrm{H}^{0}\left(E_{X_{b} / X_{0}}^{l}\right) \longrightarrow 0
$$

is exact for $l=1,2$.

2) There exists for each pair $(l, i) \quad(l=1,2,1 \leq i \leq a)$ a global section of $Q_{X_{b}}^{l}$ vanishing on $U_{j}^{l} \cap X_{b} \quad(1 \leq j<i$ if $l=1, i<j \leq a$ if $l=2)$, which gives a generator of $\left.Q_{X_{b}}^{l}\right|_{P_{i}^{l}} \cong k$. 
3) $\mathrm{H}^{1}\left(Q_{X_{b}}^{l}\right)=0$ for $l=1,2$.

Proof. We first consider the case $l=1$. 1) Easy by (3.11).

2) Since $\psi^{1}$ is locally the multiplication by $H_{i}^{1}([i / 2])(1 \leq i \leq 2 p+1)$, $H_{i}^{1}(p+1)(2 p+2 \leq i \leq 2 p+q+1)$ or $H_{i}^{1}(p)(2 p+q+2 \leq i \leq a)$ on $U_{i}^{1}(1 \leq$ $i \leq a)$ (cf. $\S 1$ ), the assertion follows from (3.11).

3) By the lemma below, we have $\mathrm{H}^{1}\left(Q_{X_{0}}^{1}\right)=0$. Hence, it follows from 1) and (2.6) that $\mathrm{H}^{1}\left(Q_{X_{b}}^{1}\right)=0$.

As for the case $l=2$, the reasoning that we have carried out is applicable to $Q_{X_{b}}^{2}$ by exchanging the roles of $\left(y_{i}, z_{i}, P_{i}^{1}, P_{i}^{2}, U_{i}^{1}, U_{i}^{2}\right)$ and $\left(z_{a-i+1}, y_{a-i+1}, P_{a-i+1}^{2}, P_{a-i+1}^{1}, U_{a-i+1}^{2}, U_{a-i+1}^{1}\right) \quad(1 \leq i \leq a)$. Consequently analogous results hold for $Q_{X_{b}}^{2}$ as well.

Lemma (3.13). (cf. $\left[8\right.$; Proposition 2]). $\mathrm{H}^{1}\left(N_{X_{0}}\right)=0$.

For each $b(0 \leq b \leq \rho(a))$ the curve $X_{b}$ is therefore flatly smoothable in $\mathbf{P}^{3}$ by (2.8). The resulting smooth curve $C$ has the neat basic sequence $\left(a ; a^{a} ; a^{b}\right)$ (see $(2.9)$ and $(3.3)$ ) and $\mathrm{H}^{1}\left(N_{C}\right)=0$. It is irreducible if and only if $0 \leq b<\rho(a)$. This proves Theorem (I. 4) in the case 1). The result is known well for $X_{0}$ (cf. [9]).

\section{§4. Curve with $B=\left(a ;(a+1)^{a} ;(a+1)^{b}\right)$}

The method of the previous section can of course be applied to the construction of curves with the other kinds of neat basic sequences, too. We will next consider curves having $\left(a ;(a+1)^{a} ;(a+1)^{b}\right)$ as their basic sequences.

Notation being as before, suppose $a \geq 2$ and let $\hat{X}_{b}$ denote the curve obtained by removing the lines $L_{j+2 p+1, j}(1 \leq j \leq q-1)$ and $L_{q+2 p+1, q+1}$ from $Y_{p}$, where $b=p(a-p)+q(0 \leq p<[a / 2], 1 \leq q \leq a-2 p-1)$.

$\operatorname{Lemma}$ (4. 1$) . \quad B\left(\hat{X}_{b}\right)=\left(a-1 ; a^{a-1} ; a^{b-1}\right)$ for all $b \quad(1 \leq b \leq \rho(a))$.

Proof. We have $X_{b-1}=\hat{X}_{b} \cup L_{q+2 p+1, q+1}$ and $B\left(X_{b-1}\right)=\left(a ; a^{a} ; a^{b-1}\right)$. Recall that the number of the lines contained in $X_{b-1}$ and passing through $P_{q+2 p+1}^{1}\left(\operatorname{resp} . P_{q+1}^{2}\right)$ is $d_{q+2 p+1}^{1}(p)\left(\operatorname{resp} . d_{q+1}^{1}(p)\right)$ (see $\left.(3.1 .1)\right)$. 
Since $h^{0}\left(\mathcal{O}_{\hat{X}_{b} \cap L_{q+2 p+1, q+1}}\right)=\left(d_{q+2 p+1}^{1}(p)-1\right)+\left(d_{q+1}^{2}(p)-1\right)=a-1$, we have the exact sequence

$$
0 \longrightarrow \mathscr{I}_{X_{b-1}}(n) \longrightarrow \mathscr{I}_{\hat{X}_{b}}(n) \longrightarrow \mathcal{O}_{L_{q+2 p+1, q+1}}(n-a+1) \longrightarrow 0
$$

(see the proof of [2; Proposition A. 1]). It follows from this that $h^{1}\left(\mathscr{I}_{\hat{X}_{b}}(n)\right)=0$ for $n \geq a-1, h^{1}\left(\mathscr{I}_{\hat{X}_{b}}(a-2)\right)=h^{1}\left(\mathscr{I}_{x_{b-1}}(a-2)\right)=b-1$ (see the proof of $(1.11)), h^{0}\left(\mathscr{I}_{\hat{x}_{b}}(a-1)\right)=1$ and $h^{0}\left(\mathscr{I}_{\hat{x}_{b}}(a)\right)=(a-1)+$ $(b-1)+4$, whence the assertion.

Lemma (4.2). The curves $\hat{X}_{b}(1 \leq b \leq \rho(a))$ are connected except for the case $a=b=4$.

Proof. Write $b=p(a-p)+q$ as above. When $p \geq 2, \hat{X}_{b}$ includes a connected curve $\left(\bigcup_{i=1}^{a} L_{i, i}\right) \cup\left(\bigcup_{i=1}^{a-2} L_{i+2, i}\right) \cup L_{q+2 p+1, q}$ which contains all the points $P_{i}^{l}(l=1,2,1 \leq i \leq a)$. Therefore $\hat{X}_{b}$ is connected. When $p=0, \hat{X}_{b}$ includes a connected curve $\left(\cup_{i=1}^{q} L_{i, i}\right) \cup\left(\cup_{i=1}^{q} L_{q+1, i}\right) \quad\left(\operatorname{resp} \cdot\left(\bigcup_{i=q+2}^{a} L_{i, i}\right) \cup\right.$ $\left.\left(\bigcup_{i=q+2}^{a} L_{i, q+1}\right)\right)$ which joins the points $P_{\imath}^{1}(1 \leq i \leq q+1)$ and $P_{\imath}^{2}(1 \leq i \leq q)$ (resp. $P_{\imath}^{1}(q+2 \leq i \leq a)$ and $\left.P_{\imath}^{2}(q+1 \leq i \leq a)\right)$, as well as the line $L_{a, 1}$ passing through $P_{a}^{1}$ and $P_{1}^{2}$. Hence $\hat{X}_{b}$ is connected. In the case where $p=1$ and $a \geq 5$, a connected curve

$$
\left(\bigcup_{i=5}^{a} L_{i, i}\right) \cup\left(\cup_{i=5}^{a} L_{i, 1}\right) \cup L_{1,1} \cup L_{3,3} \cup L_{3,1}
$$

is included in $\hat{X}_{b}$ and joins $P_{i}^{l}(l=1,2, i=1,3,5 \leq i \leq a)$. Besides, since $a-2 \geq 3$, it follows from the construction that $L_{2,2} \cup L_{a, 2} \subset \hat{X}_{b}$ and that $L_{4,4} \cup L_{4,1} \subset \hat{X}_{b}$ or $L_{4,4} \cup L_{4,2} \subset \hat{X}_{b}$. The points $P_{\imath}^{l}(l=1,2, i=2,4)$ therefore lie on the connected component of $\hat{X}_{b}$ containing $P_{1}^{1}$, which implies the assertion. The remaining case is, in fact, the exceptional one, i. e. $a=b=4$, and we see directly that $\hat{X}_{4}(a=4)$ is the disjoint union of $L_{2,2}$ and $L_{1,1} \cup L_{3,3} \cup L_{4,4} \cup L_{3,1} \cup L_{4,1}$.

Set

$$
\begin{cases}\hat{H}_{i}^{1}(p+1) & =H_{i}^{1}(p) z_{i-2 p} / x_{1} \quad(0 \leq p \leq[(i-1) / 2]) \\ \hat{H}_{i}^{2}(p+1) & =H_{i}^{2}(p) y_{i+2 p} / x_{2} \quad(0 \leq p \leq[(a-i) / 2]), \\ \hat{G}_{i}^{l}(p+1) & =G_{i}^{l}(0) / \hat{H}_{i}^{l}(p+1)\end{cases}
$$

and $\hat{E}_{i}^{l}(p+1)=c_{P_{i}^{l} *}\left(k\left[U_{i}^{l}\right] /\left(F_{i}^{l}, \hat{G}_{i}^{l}(p+1), \hat{H}_{l}^{i}(p+1)\right)\right)(l=1,2)$ for $1 \leq i$ $\leq a$. Then 


$$
\left\{\begin{array}{l}
E_{\hat{X}_{b} / X_{0}}^{1}=\left(E_{X_{b} / X_{0}}^{1} \oplus \hat{E}_{2 p+q+1}^{1}(p+1)\right) / E_{2 p+q+1}^{1}(p+1) \\
E_{\hat{X}_{b} / X_{0}}^{2}=\left(E_{X_{b} / X_{0}}^{2} \oplus E_{q}^{2}(p) \oplus \hat{E}_{q+1}^{2}(p+1)\right) /\left(E_{q}^{2}(p+1) \oplus E_{q+1}^{2}(p)\right)
\end{array} .\right.
$$

We have the following result similar to 1) of (3.11) concerning $F_{i}^{1}, \hat{G}_{i}^{1}(p+1)$ and $\hat{H}_{i}^{1}(p+1)$.

Proposition (4.3). Let $\zeta$ be an element of $k\left[x_{2} / x_{1}, x_{3} / x_{1}, x_{4} / x_{1}\right]$. There exist, for each pair $(i, p) \quad(1 \leq i \leq a, 0 \leq p \leq[(i-1) / 2]$, pre-normals $v$,w of $Y_{p}$ satisfying $\pi_{\nu}^{1}(v)=\pi_{\nu}^{1}(w)=0 \quad(1 \leq \nu \leq i-1)$ such that

$$
\left\{\begin{array}{l}
\zeta-\pi_{i}^{1}(v) \equiv 0\left(\bmod \left(F_{i}^{1}, \hat{G}_{i}^{1}(p+1), \hat{H}_{i}^{1}(p+1)\right)\right) \\
\pi_{i}^{1}(w) \equiv \hat{H}_{i}^{1}(p+1)\left(\bmod F_{i}^{1}\right)
\end{array}\right.
$$

Proof. Entirely similar to the proof of (3.11). For $i=a$, use (3.9) and (2.15).

The assertions of $(3.12)$ are therefore valid for $\hat{X}_{b}$ also. Thus the reasoning above gives a proof of Theorem (I.4) for the case 2), unless $a=b=3$. Let $X$ be the curve obtained by removing $L_{3,2}, L_{4,3}$ and $L_{4,1}$ from $\operatorname{Proj}\left(R /\left(y_{2} y_{3} y_{4}, z_{1} z_{2} y_{3} y_{4}, z_{1} z_{2} z_{3} y_{4}, z_{1} z_{2} z_{3} z_{4}\right)\right)$. One checks easily that $B(X)=\left(3 ; 4^{3} ; 4^{3}\right)$ and that $X$ is a connected union of six lines, each of which meets the others in at most two points. Hence by [10; Corollary 4.2] the theorem holds for this case as well.

Remark (4.4). Let $b=p(a-p)+q \geq 2$ be as in the beginning of this section. Eliminate $L_{1,1}$ from $X_{b} \cup L_{2,1}$ and denote the resulting curve by $X_{b}^{\prime}$. One sees that $B\left(X_{b}^{\prime}\right)=\left(a-1 ; a^{a-1} ; a^{b-1}\right)$ and that $X_{b}^{\prime}$ are connected. The curves $X_{b}^{\prime}$ are smoothable with $\mathrm{H}^{1}\left(N_{X_{b}^{\prime}}\right)=0$, so they also serve for a proof of (I.4) in the case 2). We have adopted $\hat{X}_{b}$ to make the argument as parallel as possible with the one carried out in the preceding section.

\section{§5. Curve with $\mathbb{B}=\left(a ; a^{a} ;(a+1)^{b}\right)$}

In this and the next sections, the notation defined previously will sometimes be altered.

Let $a \geq 1, J=\left(f_{0}, f_{1}, \ldots, f_{a}\right) R, A$ and $X_{0}=\operatorname{Proj} R / J=Z(A)$ be as in section 3. We define $A(b)$ and $X_{b}$ anew by 
$\left\{\begin{array}{l}A(b)=A \backslash(\{(j+2 l, j) \mid 1 \leq j \leq a-2 l, 1 \leq l \leq p\} \cup\{(j+2 p+2, j) \mid 1 \leq j \leq q\}) \\ X_{b}=Z(A(b))\end{array}\right.$ for $b=p(a-p-1)+q(1 \leq q \leq a-2 p-2, \quad 0 \leq p<[(a-1) / 2])$, and for each $p(0 \leq p \leq[(a-1) / 2])$ we set $Y_{p}=X_{p(a-p-1)}$. Recall that $p(a-p-1)$ $=p^{\prime}\left(a-p^{\prime}-1\right)+q^{\prime}$ with $p^{\prime}=p-1, q^{\prime}=a-2 p^{\prime}-2$ for $p>0$. Clearly $X_{b}(1 \leq b \leq \rho(a-1))$ is connected and $B\left(X_{b}\right)=\left(a ; a^{a} ;(a+1)^{b}\right)$. For an integer $l$, we denote by $Y_{p}^{l}$ the curve $Y_{p}$ defined as above with $a$ replaced by $l$. If a polynomial $f$ of $R$ is homogeneous in both $\left(x_{1}, x_{3}\right)$ and $\left(x_{2}, x_{4}\right)$ of degree $m$ and $m^{\prime}$ respectively, we will say that it is homogeneous of bidegree $\left(m, m^{\prime}\right)$.

Lemma (5。1). Let $m$ be a nonnegative integer. For each $c(0 \leq c \leq m)$, there is a homogeneous polynomial of bidegree $(m, m+1)$ which vanishes on $Y_{m}^{2 m+1} \backslash L_{2 c+1,2 c+1}$ but not on $L_{2 c+1,2 c+1}$.

Proof. We proceed by induction on $m$. Set $e=\left(\prod_{l=1}^{m} y_{2 l} z_{2 l}\right) z_{\text {. For }}$ $m=0$, put $z=x_{2}$. If $m \geq 1$, put $z=z_{1}$ or $z=z_{2 m+1}$. In the first case $e$ becomes zero on $Y_{m}^{2 m+1} \backslash \bigcup_{l=1}^{m} L_{2 l+1,2 l+1}$ but not on $L_{2 m+1,2 m+1}$, while in the second case it vanishes on $Y_{m}^{2 m+1} \backslash \bigcup_{l=0}^{m-1} L_{2 l+1,2 l+1}$ but not on $L_{1,1}$. This gives a. proof for $m=1$. If $m \geq 2$, the curve $Y:=Y_{m}^{2 m+1} \cap\left(\underset{3 \leq j \leq i \leq 2 m-1}{\cup} \mathbb{L}_{i, j}\right)$ may be thought of as a copy of $Y_{m-2}^{2 m-3}$. The induction hypothesis therefore implies that for each $c(1 \leq c \leq m-1)$ there is a homogeneous polynomial of bidegree $(m-2, m-1)$ which vanishes on $Y \backslash L_{2 c+1,2 c+1}$ but not on $L_{2 c+1,2 c+1}$. Multiply it by $z_{1} z_{2} y_{2 m} y_{2 m+1}$, and we get a desired polynomial for $1 \leq c \leq m-1$. In the case $c=0$ or $c=m$, take a suitable linear combination of $e$ and the polynomials thus obtained.

Corollary (5.2). Set $e_{m}=y_{1}\left(\prod_{l=1}^{m} y_{2 l} z_{2 l}\right) z_{2 m+1}(m \geq 0)$. For each pair $(m, n) \quad(n \geq 2 m+1 \geq 1)$, there exists a homogeneous polynomial $e_{m, n}$ of bidegree $(m+1, m+1)$ which vanishes on $Y_{m}^{2 m+1}$, such that

$$
e_{m, n} \equiv e_{m}\left(\bmod y_{2 m} y_{2 m+1} y_{n}\right) \text { 。 }
$$

Proof. One verifies directly that $e_{m} \equiv 0$ on $Y_{m}^{2 m+1} \backslash \bigcup_{l=1}^{m-1} L_{2 l+1,2 l+1}$. If $m=0,1$, we are done. In the case $m \geq 2$, since $Y=Y_{m}^{2 m+1} \cap\left(\underset{3 \leq j \leq i \leq 2 m-1}{\cup} \mathbb{L}_{i, j}\right)$ 
is a copy of $Y_{m-2}^{2 m-3}$, there are homogeneous polynomials $e_{c}^{\prime}(1 \leq c \leq m-1)$ of bidegree $(m-2, m-1)$ such that $e_{c}^{\prime} \equiv 0$ on $Y \backslash L_{2 c+1,2 c+1}$ and $e_{c}^{\prime} \not \equiv 0$ on $L_{2 c+1,2 c+1}$ by $(5.1)$. Besides, the bidegrees of $e_{m}$ and $z_{1} z_{2} e_{c}^{\prime} y_{2 m} y_{2 m+1} y_{n}$ $(1 \leq c \leq m-1)$ coincide, therefore $e_{m, n}$ can be obtained as a linear combination of them.

Proposition (5.3). For each triple $(p, q, m)$ satisfying $0 \leq m \leq p \leq$ $[(a-1) / 2], 0 \leq q \leq a-2 m-1$, there exists a homogeneous polynomial of degree $a+1$ vanishing on $Y_{p}$ which is congruent to

$$
\left(\prod_{l=1}^{q} z_{l}\right) y_{q+1}\left(\prod_{l=1}^{m} y_{q+2 l} z_{q+2 l}\right) z_{q+2 m+1}\left(\prod_{l=q+2 m+2}^{a} y_{l}\right)
$$

modulo $y_{a}\left(\prod_{l=q+2 m}^{a} y_{l}\right)$.

Proof. Note that $Y_{m} \supset Y_{p}$. Since $Y^{\prime}:=Y_{m} \cap \underset{q+1 \leq j \leq i \leq q+2 m+1}{\cup} L_{i, j}$ is a copy of $Y_{m}^{2 m+1}$, there exists by (5.2) a homogeneous polynomial of degree $2 m+2$ vanishing on $Y^{\prime}$ and congruent to $y_{q+1}\left(\prod_{l=1}^{m} y_{q+2 l} z_{q+2 l}\right) z_{q+2 m+1}$ modulo $y_{q+2 m} y_{q+2 m+1} y_{a}$. It is enough to multiply it by $\left(\prod_{l=1}^{q} z_{l}\right)\left(\prod_{l=q+2 m+2}^{a} y_{l}\right)$ to get a desired polynomial.

This time, put

$$
\left\{\begin{array}{ll}
H_{i}^{1}(p)=\left(\prod_{l=1}^{p} z_{i-2 l}\right) / x_{1}^{p} & (0 \leq p \leq[(i-1) / 2]) \\
H_{i}^{2}(p)=\left(\prod_{l=1}^{p} y_{i+2 l}\right) / x_{2}^{p} & (0 \leq p \leq[(a-i) / 2])
\end{array} .\right.
$$

The definitions of $D_{l}(p), W_{l}(p)(0 \leq p \leq[(a-1) / 2], l \geq 1)$ and $G_{i}^{l}(p)$, $E_{i}^{l}(p) \quad(l=1,0 \leq p \leq[(i-1) / 2]$ or $l=2,0 \leq p \leq[(a-i) / 2])$ are exactly the same as in section 3 . We have

$$
\left\{\begin{array}{l}
E_{X_{b} / X_{0}}^{1}=\left(\bigoplus_{i=1}^{2 p+2} E_{i}^{1}([(i-1) / 2])\right) \oplus\left(\bigoplus_{i=2 p+3}^{2 p+q+2} E_{i}^{1}(p+1)\right) \oplus\left(\bigoplus_{i=2 p+q+3}^{a} E_{i}^{1}(p)\right) \\
\left.E_{X_{b} / X_{0}}^{2}=\left(\bigoplus_{j=1}^{q} E_{j}^{2}(p+1)\right) \oplus\left(\bigoplus_{j=q+1}^{a-2 p-2} E_{j}^{2}(p)\right) \oplus\left(\bigoplus_{j=a-2 p-1}^{a} E_{j}^{2}[(a-j) / 2]\right)\right)
\end{array},\right.
$$

where $b=p(a-p-1)+q(1 \leq q \leq a-2 p-2,0 \leq p<[(a-1) / 2])$.

Lemma (5.4). Let $p, r, n$ be integers satisfying $r \geq 0,2 n-r \leq a$, $0 \leq n-r-1 \leq p \leq[(a-1) / 2]$. Then $g_{r}^{(n-1)} u_{2 n-r-1} \in W_{a+1}(p)$. 
Proof. Apply (5.3) with $q=r, m=n-r-1$.

The rest of the proof for the smoothability of $X_{b}$ is entirely similar to the previous two cases. We have thus proved Theorem (I.4) in the case 3 ).

$$
\begin{gathered}
\text { \$6. Curve with } \mathbb{B}=\left(a ; a^{c},(a+1)^{a-c} ;(a+1)^{b}\right) \\
\text { or } B=\left(a ; a^{a} ; \mathfrak{a}^{e},(a+1)^{b-e}\right)
\end{gathered}
$$

To avoid too much complexity, we will restrict our consideration to the case where $0 \leq b \leq a-2$.

Let $a, a^{\prime}, b, b^{\prime}$ be integers such that $0 \leq b \leq a-2,1 \leq a^{\prime} \leq a-2,1 \leq b^{\prime} \leq$ $b-1, A^{\prime}$ the set $A \backslash\left\{\left(i, a-a^{\prime}\right) \mid a-a^{\prime} \leq i \leq a\right\}$ and $J^{\prime}$ the ideal in $R$ generated by $f_{i}\left(0 \leq i \leq a-a^{\prime}-1\right)$ and $f_{i} / z_{a-a^{\prime}}\left(a-a^{\prime} \leq i \leq a\right)$. Denote the curve

$$
Z\left(A \backslash\left(\left\{(j+1, j) \mid 1 \leq j \leq b^{\prime}\right\} \cup\left\{(j+2, j) \mid b^{\prime}+1 \leq j \leq b\right\}\right)\right)
$$

by $X$, and if $0 \leq b \leq a-3$, define $X_{b}^{\prime}$ by

$$
X_{b}^{\prime}=\left\{\begin{array}{l}
Z\left(A^{\prime} \backslash\{(j+1, j) \mid 1 \leq j \leq b\}\right) \quad\left(0 \leq b \leq a-a^{\prime}-2\right) \\
Z\left(A^{\prime} \backslash\left(\begin{array}{l}
\left\{(j+1, j) \mid 1 \leq j \leq a-a^{\prime}-2\right\} \\
\cup\left\{(j+2, j) \mid a-a^{\prime}-1 \leq j \leq b+1, j \neq a-a^{\prime}\right\}
\end{array}\right)\right) \\
\left(a-a^{\prime}-1 \leq b \leq a-3\right)
\end{array}\right.
$$

Note that $X_{0}^{\prime}=Z\left(A^{\prime}\right)=\operatorname{Proj} R / J^{\prime}$ and $B\left(X_{0}^{\prime}\right)=\left(a-1 ;(a-1)^{a^{\prime}}, a^{a-a^{\prime}-1}\right)$. Both $X_{b}^{\prime}$ and $X$ are connected and $B\left(X_{b}^{\prime}\right)=\left(a-1 ;(a-1)^{a^{\prime}}, a^{a-a^{\prime}-1} ; a^{b}\right)$, $B(X)=\left(a ; a^{a} ; a^{b^{\prime}}(a+1)^{b-b^{\prime}}\right)$ by (3.2). The smoothability of $X$ follows from (3.11), (3.12) and the consequences of (5.4) similar to them. As for $X_{b}^{\prime}$, we had better make some more points clear. Assume $a \geq 4$. Put $D_{l}^{\prime}(p)=I_{x_{p}^{\prime}, l} \cap y_{a} R$ and $W_{l}^{\prime}(p)=\left\{f / y_{a} x_{1}^{l-1}\left(\bmod F_{a}^{1}\right) \mid\right.$ $\left.f \in D_{l}^{\prime}(p)\right\} \subset k[s, t]$ for $l \geq 1, p=0,1$.

Lemma (6.1). 1) $\prod_{l=1}^{i} u_{l} \in W_{a}^{\prime}(0) \quad$ for $0 \leq i \leq a-a^{\prime}-1$

and

$$
\left(\prod_{l=1}^{i} u_{l}\right) / u_{a-a^{\prime}} \in W_{a-1}^{\prime}(0) \quad \text { for } a-a^{\prime} \leq i \leq a-1 \text {. }
$$

2) $k[s, t]_{1} \subset W_{a}^{\prime}(1)$. 
Proof. 1) Clear by $f_{i} \in D_{a}^{\prime}(0) \quad\left(0 \leq i \leq a-a^{\prime}-1\right)$ and $f_{i} / z_{a-a^{\prime}} \in$ $D_{a-1}^{\prime}(0) \quad\left(a-a^{\prime} \leq i \leq a-1\right)$.

2) We have $u_{1} \in W_{a}^{\prime}(0)$ by 1$)$. When $3 \leq a-a^{\prime} \leq a-1, I_{x_{1}^{\prime}, a}$ contains $y_{1} z_{2}\left(\prod_{l=3}^{a} y_{l}\right)$, so $u_{2} \in W_{a}^{\prime}(1)$. On the other hand, if $a-a^{\prime}=2$, we have $y_{1} y_{2} z_{3}\left(\prod_{l=4}^{a} y_{l}\right) \in I_{x_{1}^{\prime}, a}$ and $u_{3} \in W_{a}^{\prime}(1)$. Hence $s, t \in W_{a}^{\prime}(1)$.

We set $H_{i}^{1}=u_{a-a^{\prime}} u_{i-2}, G_{i}^{\prime 1}=\left(\prod_{l=1}^{i} u_{l}\right) / H_{i}^{1}\left(a-a^{\prime}+1 \leq i \leq a\right)$.

Lemma (6. 2). Assume $a^{\prime} \neq 2$. 1) There exists for each $\xi \in k[s, t] a$ pre-normal $v^{1}(\xi)$ of $X_{0}^{\prime}$ such that $v_{\nu}^{1}(\xi)=0(0 \leq \nu \leq a-1)$ and $v_{a}^{1}(\xi) / x_{1}^{a} \equiv \xi$ $\left(\bmod \left(F_{a}^{1}, G_{a}^{\prime 1}, H_{a}^{1}\right)\right)$.

2) There exists a pre-normal $w^{1}$ of $X_{1}^{\prime}$ such that $w_{\nu}^{1}=0 \quad(0 \leq \nu \leq a-1)$ and $w_{a}^{1} / x_{1}^{a} \equiv H_{a}^{1}\left(\bmod F_{a}^{1}\right)$ 。

Proof. Easy by (6.1) and $(2.14)$.

Since the stalk of $E_{X_{b}^{\prime} / X_{0}}^{l}$ at $P_{i}^{l}(l=1,2,1 \leq i \leq a)$ is isomorphic to

$$
\begin{aligned}
& k\left[U_{i}^{1}\right] /\left(F_{i}^{1}, G_{i}^{\prime 1}, H_{i}^{1}\right) \quad\left(a-a^{\prime}+1 \leq i \leq a, i \neq a-a^{\prime}+2\right), \\
& k\left[U_{i}^{1}\right] /\left(F_{i}^{1}, u_{a-a^{\prime}},\left(\prod_{l=1}^{i} u_{l}\right) / u_{a-a^{\prime}}\right) \quad\left(a-a^{\prime} \leq i \leq a\right)
\end{aligned}
$$

or one of the corresponding stalks of $E_{Y_{1} / X_{0}}^{l}$ which appeared in Sections 3 and 5 , we find by (3.11), (4.3), (5.4), (6.1), (6.2) and their consequences that $X_{b}^{\prime}$ is smoothable for $0 \leq b \leq a-3$. The result is known well for $X_{0}^{\prime}$ (cf. [9]). This completes the proof of Theorem (I.4).

Corollary (6.3)。 For each $g \geq 0$ the neat basic sequence $B_{\langle g\rangle}$ is realised by a smooth irreducible curve such that the cohomology of its normal bundle vanishes in degree one.

Proof. Obvious by (I. 4$)$, since $0 \leq b\left(B_{\langle g\rangle}\right) \leq a\left(B_{\langle g\rangle}\right)-2$ (see Section 1)。 


\section{\$7. Concluding Remarks}

\section{Stick Figures}

The reduced singular curves which have been considered so far can be deformed into smoothable stick figures (see $[10 ; \S 6]$ ) as we will explain below. Given a positive integer $a$, let $\zeta_{i 3}, \zeta_{i 4}, \xi_{i 3}, \xi_{i 4}(1 \leq$ $i \leq a)$ be parameters and set

$$
\tilde{y}_{i}=x_{1}-\zeta_{i 3} x_{3}-\zeta_{i 4} x_{4}, \quad \tilde{z}_{i}=x_{2}-\xi_{i 3} x_{3}-\xi_{i 4} x_{4}
$$

for $1 \leq i \leq a$. Denote the parameter space Spec $k\left[\zeta_{i 3}, \zeta_{i 4}, \xi_{i 3}, \xi_{i 4} ; 1 \leq i \leq a\right]$ by $T$. For each pair $(i, j) \quad(1 \leq i \leq a, 1 \leq j \leq a)$ the scheme

$$
\tilde{L}_{i, j}:=\operatorname{Proj}\left(R \otimes_{k} k[T] /\left(\tilde{y}_{i}, \tilde{z}_{j}\right)\right) \subset \mathbb{P}_{T}^{3}
$$

is a flat family of lines over $T$. Put

$$
\begin{gathered}
V=\left\{v \in T \mid \begin{array}{l}
\left(\tilde{y}_{i_{1}}, \tilde{y}_{i_{2}}, \tilde{z}_{i_{1}}, \tilde{z}_{j_{2}}\right) R \otimes_{k} k(v)=\left(x_{1}, x_{2}, x_{3}, x_{4}\right) R \otimes_{k} k(v) \\
\text { for all } 1 \leq i_{1}<i_{2} \leq a, \quad 1 \leq j_{1}<j_{2} \leq a
\end{array}\right\}, \\
V_{1}=\left\{v \in V \mid \begin{array}{l}
\operatorname{dim}_{k(v)}\left(\tilde{y}_{i_{1}}, \tilde{y}_{i 2}, \tilde{y}_{i 3}\right) k(v)=\operatorname{dim}_{k(v)}\left(\tilde{z}_{i_{1}}, \tilde{z}_{i_{2}}, \tilde{z}_{i_{3}}\right) k(v) \\
=3 \text { for all } 1 \leq i_{1}<i_{2}<i_{3} \leq a
\end{array}\right\} .
\end{gathered}
$$

Given a subset $A$ of $\{(i, j) \mid 1 \leq i \leq a, 1 \leq j \leq a\}$, we consider the schemetheoretic union $\tilde{Z}(A):=\left(\bigcup_{(i, j) \in A} \tilde{L}_{i, j}\right) \cap \mathbb{P}_{V}^{3}$ of $\tilde{L}_{i j} \cap \mathbb{P}_{V}^{3}$ as a family of singular curves over $V$. Note that the fiber $\tilde{Z}(A)$ 。 over the point $o$ defined by $\zeta_{i 3}=\alpha_{i}, \zeta_{i 4}=\xi_{i 3}=0, \xi_{i 4}=\beta_{i}(1 \leq i \leq a)$ coincides with $Z(A)$. Since $\tilde{Z}(A)$ is contained in the surfaces $\prod_{l=1}^{a} \tilde{y}_{l}=0$ and $\prod_{l=1}^{a} \tilde{z}_{l}=0$, it follows from the definition of $V$ that $\tilde{Z}(A)$ is given locally by a system of equations of the form

$$
\tilde{y}_{i} / x=0, \tilde{z}_{i_{1}} \cdot \tilde{z}_{i_{2}} \cdots \tilde{z}_{i_{r}} / x^{r}=0
$$

or

$$
\tilde{z}_{i} / x=0, \tilde{y}_{i_{1}} \cdot \tilde{y}_{i_{2}} \cdots \tilde{y}_{i r} / x^{r}=0,
$$

where $1 \leq i \leq a, i_{1}<i_{2}<\cdots<i_{r}(r \geq 1)$ and $x$ is a suitable linear form of $x_{1}, x_{2}, x_{3}, x_{4}$. The family $\tilde{Z}(A)$ is therefore flat over $V$ and the fibers over the points of $V_{1}$ are all stick figures.

Proposition (7.1). The neat basic sequences listed in (I.4) are 
realised by strongly smoothable stick figures such that the cohomologies of their normal sheaves vanish in degree one.

Proof. Apply the above method to the singular curves appeared in Sections 3, 4,5 and 6. Since Hilb $\left(\mathbf{P}^{3}\right)$ is locally irreducible at the points corresponding to them, the resulting stick figures are also smoothable.

Corollary (7.2) (cf. [10; Propositions 6. 1, 6.2,6.3 and 6.4]). For each pair of integers $(d, g)$ satisfying $g \geq 0, d \geq \operatorname{deg}\left(B_{\langle g\rangle}\right)$, there is a strongly smoothable stick figure with degree $d$ and genus $g$.

Proof. Notice first that $B_{\langle g\rangle}$ is included in the list of (I. 4). Attach lines successively to a strongly smoothable stick figure which has the basic sequence $B_{\langle g\rangle}$, with at most two intersection points at a time (cf. [10 ; Corollary 4.2]).

It will be interesting if we can prove that every neat basic sequence with positive degree and nonnegative genus is realised by a smoothable stick figure.

\section{Deficiency Modules}

Next, let us look a little bit into the deficiency module of each smooth irreducible curve $C$ we have constructed. It is desirable that the equality

$$
h^{1}\left(\mathscr{I}_{C}(n)\right)=\max \left(1-g+d n-\left(\begin{array}{c}
n+3 \\
3
\end{array}\right), 0\right)
$$

( $d=$ degree, $g=$ genus) should hold for all $n \geq 0$, but this cannot be checked instantly, because unfortunately the singular curves used in the proof of (I.4) do not necessarily satisfy the same equality beforehand. In the cases $B=\left(a ; a^{a} ; a^{b}\right) \quad(0 \leq b \leq a-1), B=\left(a ;(a+1)^{a}\right.$; $\left.(a+1)^{b}\right)(0 \leq b \leq a), B=\left(a ; a^{c},(a+1)^{a-c} ;(a+1)^{b}\right)(1 \leq c \leq a-1, c$ is small, $0 \leq b \leq a-2), B=\left(a ; a^{a} ; a^{c},(a+1)^{b-c}\right) \quad(1 \leq c \leq b-1, b-c$ is small, $0 \leq b$ $\leq a-2)$ and in the case $0 \leq b \leq 3$, one can verify (7.3) by deforming the free resolutions of the homogeneous ideals of the singular curves. We will see this carefully especially in the first case. Let 


$$
a, \bar{n}^{1}=\left(n_{1}, \ldots, n_{a}\right) \text { and } \bar{n}^{2}=\left(n_{a+1}, \ldots, n_{a+b}\right)
$$

denote the head, the body and the tail of $B:=B(C)$ respectively and let $X_{\text {sing }}$ denote the reduced singular curve such that $B\left(X_{\text {sing }}\right)=B$, which we have considered. Then $I_{X_{\text {sing }}}$ has a minimal free resolution of the form

$$
\begin{gathered}
0 \longrightarrow R\left[-\bar{n}^{2}-2\right] \stackrel{\lambda_{3}^{*}}{\longrightarrow} R\left[-\bar{n}^{1}-1,-\bar{n}^{2}-1,-\bar{n}^{2}-1\right] \\
\stackrel{\lambda_{2}^{*}}{\longrightarrow} R\left[-a,-\bar{n}^{1},-\bar{n}^{2}\right] \stackrel{\lambda_{1}^{*}}{\longrightarrow} I_{X_{\text {sing }}} \longrightarrow 0
\end{gathered}
$$

(cf. [2; Notation, p. 802]). By Serre duality, $M^{\prime}\left(X_{\text {sing }}\right)_{-n}$ is isomorphic to the cokernel of the map

$$
\chi_{n}: R\left[\bar{n}^{1}-3, \bar{n}^{2}-3, \bar{n}^{2}-3\right]_{-n} \stackrel{{ }^{t} \lambda_{3}^{*}}{\longrightarrow} R\left[\bar{n}^{2}-2\right]_{-n} \text { 。 }
$$

With the help of this, the case $B=\left(a ; a^{a} ; a^{b}\right)(0 \leq b \leq a-1)$ can be treated as follows. We have

$$
\begin{aligned}
& \lambda_{1}^{*}=\left(f_{0}^{*}, f_{1}^{*}, \ldots, f_{a}^{*}, f_{a+1}^{*}, \ldots, f_{a+b}^{*}\right), \lambda_{2}^{*}=\left[\begin{array}{ccc}
U_{01}^{*} & U_{02}^{*} & 0 \\
U_{1}^{*} & U_{2}^{*} & U_{4}^{*} \\
0 & U_{3}^{*} & U_{5}^{*}
\end{array}\right], \\
& \lambda_{3}^{*}=\left[\begin{array}{r}
U_{6}^{*} \\
-U_{5}^{*} \\
U_{3}^{*}
\end{array}\right] \text {, with } f_{i}^{*}=\left(\prod_{l=1}^{i} z_{l}\right)\left(\prod_{l=i+1}^{a} y_{l}\right) \quad(0 \leq i \leq a), \\
& f_{a+j}^{*}=\left(\prod_{l=1}^{j-1} z_{l}\right) y_{j} z_{j+1}\left(\prod_{l=j+2}^{a} y_{l}\right) \quad(1 \leq j \leq b), \\
& U_{3}^{*}=D\left(y_{2}, y_{3}, \ldots, y_{b+1}\right), U_{5}^{*}=D\left(z_{1}, z_{2}, \ldots, z_{b}\right),
\end{aligned}
$$

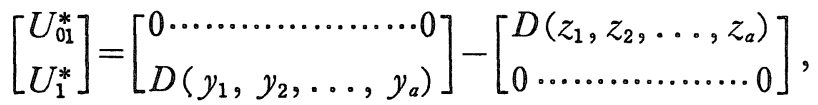

$$
\begin{aligned}
& {\left[\begin{array}{c}
U_{02}^{*} \\
U_{2}^{*}
\end{array}\right]=-\left[\begin{array}{c}
D\left(z_{2}, z_{3}, \ldots, z_{b+1}\right) \\
0
\end{array}\right], U_{4}^{*}=-\left[\begin{array}{c}
0 \ldots \ldots \ldots \ldots \ldots . .0 \\
D\left(y_{1}, y_{2}, \ldots, y_{b}\right) \\
0
\end{array}\right],} \\
& U_{6}^{*}=\left[\begin{array}{ccccc}
z_{2} & & & & 0 \\
y_{1} & z_{3} & & & \\
& y_{2} & \ddots & & \\
& & & \ddots & \\
& & & & z_{b+1} \\
& 0 & & & y_{b} \\
& & 0 &
\end{array}\right],
\end{aligned}
$$


where $D\left(c_{1}, c_{2}, \ldots, c_{n}\right)$ denotes a diagonal matrix with components $c_{1}, c_{2}, \ldots, c_{n}$ in its diagonal. Note that these matrices are not normalised into the forms described in $[2 ; \S 1]$. For $n=a-2, a-3, a-4$, the function $-K_{B}(n)=1-g+d n-\left(\begin{array}{c}n+3 \\ 3\end{array}\right)$ (cf. (1.8)) takes the values $b$, $2 b-a, 3 b-3 a+1$, so that the expected dimensions of $\mathrm{H}^{1}\left(\mathscr{I}_{c}(n)\right)$ are $b, \max (2 b-a, 0), 0$ in order. On the other hand, direct computations of $\operatorname{Im}\left(\chi_{n}\right)$ show that

$$
h^{1}\left(\mathscr{I}_{X_{\text {sing }}}(n)\right)=\left\{\begin{array}{ll}
b & (n=a-2) \\
b-1 & (n=a-3) \\
0 & (n \leq a-4, n \geq a-1)
\end{array},\right.
$$

therefore (7.3) is not true for all $n \geq 0$, if $1<b<a-1$.

Lemma (7.5)。 With the notation above, suppose that $\chi_{m}$ is surjective and that the rows of $\lambda_{2}^{*}$ lie in $\underset{n \leq m}{\bigoplus} R\left[\bar{n}^{1}-3, \bar{n}^{2}-3, \bar{n}^{2}-3\right]_{-n}$, for some $m \geq a-4$. Then for any matrix $\lambda$ of the same size as $\lambda_{3}^{*}$ with components in the set of homogeneous polynomials of $R$, which satisfies $\Delta(\lambda)=\Delta\left(\lambda_{3}^{*}\right)$ (cf. $[2$; Notation]), the exact sequence (7.4) has a lifting

$$
\begin{aligned}
0 \longrightarrow & \tilde{R}\left[-\bar{n}^{2}-2\right] \stackrel{\tilde{\lambda}_{3}^{*}}{\longrightarrow} \tilde{R}\left[-\bar{n}^{1}-1,-\bar{n}^{2}-1,-\bar{n}^{2}-1\right] \\
& \stackrel{\tilde{\lambda}_{2}^{*}}{\longrightarrow} \tilde{R}\left[-a,-\bar{n}^{1},-\tilde{n}^{2}\right] \stackrel{\tilde{\lambda}_{1}^{*}}{\longrightarrow} \tilde{I} \longrightarrow 0,
\end{aligned}
$$

such that $\tilde{\lambda}_{3}^{*}-\lambda_{3}^{*}=\zeta \lambda$, where $\zeta$ is a parameter, $\tilde{R}:=R \otimes_{k} k[[\zeta]]$ and $\tilde{I}$ is an ideal in $\tilde{R}$.

Proof. Put $\tilde{\lambda}_{3}^{*}=\lambda_{3}^{*}+\zeta \lambda$. Since the rows of $\lambda_{2}^{*}$ lie in $\underset{n \leq m}{\bigoplus} R\left[\tilde{n}^{1}-3\right.$, $\left.\bar{n}^{2}-3, \bar{n}^{2}-3\right]_{-n}$ by hypothesis, there is a matrix $\tilde{\lambda}_{2}^{*}$ of the same size as $\lambda_{2}^{*}$ whose components are homogeneous polynomials in $x_{1}, x_{2}, x_{3}, x_{4}$ over $k[[\zeta]]$ and which satisfies $\tilde{\lambda}_{2}^{*} \cdot \tilde{\lambda}_{3}^{*}=0, \Delta\left(\tilde{\lambda}_{2}^{*}\right)=\Delta\left(\lambda_{2}^{*}\right)$ and $\tilde{\lambda}_{2}^{*} \equiv \lambda_{2}^{*}$ $(\bmod \zeta \tilde{R})$. Using $\tilde{\lambda}_{2}^{*}$, define $\tilde{\lambda}_{1}^{*}$ by the formula in $[2 ; \operatorname{Remark} 1.8]$ (cf. $[6$; Theorem 3.1 (a) $]$ ).

This lemma implies the existence of a flat family $\operatorname{Proj}_{k[[\zeta]]}(\tilde{R} / \tilde{I})$ over Spec $k[[\zeta]]$. In the present case, we conclude therefore that (7.3) is true for all $n \geq 0$, since $\mathrm{Hilb}\left(\mathbf{P}^{3}\right)$ is irreducible in a neighborhood of the point corresponding to $X_{\text {sing }}$. Furthermore, we may assume by the above lemma that 


$$
M^{\prime}(C) \cong R[a-2]^{a} / \operatorname{Im}^{R}(W) \quad(\mathrm{cf} .[2 ; \text { Notation }])
$$

with a generic $b \times(a+2 b)$-matrix $W(1 \leq b \leq a-1)$ whose components are linear forms in $x_{1}, x_{2}, x_{3}, x_{4}$. The remaining cases mentioned above can be dealt with almost similarly. It seems, however, difficult to apply this method to all the other cases and we have not checked (7.3) fully yet.

\section{Appendix. Basic Sequence and Deficiency Module}

Let $M$ be a graded $R$-module of finite length. If the structure of $M$ is thoroughly understood, it is rather easy to construct a curve $X$ which has a basic sequence given beforehand, such that $M(X) \cong M$ up to shift in grading. Suppose that $M$ has a minimal free resolution of the form

$$
0 \longrightarrow S\left[-\bar{\varepsilon}^{2}\right] \stackrel{G}{\longrightarrow} S\left[-\bar{\varepsilon}^{1}\right] \stackrel{H}{\longrightarrow} S\left[-\bar{\varepsilon}^{0}\right] \longrightarrow M \longrightarrow 0
$$

as an $S$-module, where $\bar{\varepsilon}^{i}=\left(\varepsilon_{1}^{i}, \ldots, \varepsilon_{r_{2}}^{i}\right) \quad(i=0,1), \bar{\varepsilon}^{2}=\left(\varepsilon_{1}^{2}, \ldots, \varepsilon_{b}^{2}\right), 0<\varepsilon_{1}^{2}$ $\leq \cdots \leq \varepsilon_{b}^{2}$ and $x_{1}, x_{2}, x_{3}, x_{4}$ are chosen generically. Given a positive integer $a$ and a sequence of integers $\bar{n}^{1}=\left(n_{1}, \ldots, n_{a}\right)$ such that $a \leq n_{1} \leq \cdots \leq n_{a}$, we set $B=\left(a ; \bar{n}^{1}, \bar{\varepsilon}^{2}\right)$. We will assume that

$$
\text { (A. 2) } \quad\left(\bar{n}^{1}+1, \bar{\varepsilon}^{0}+2, \bar{\varepsilon}^{1}+1, \bar{\varepsilon}^{1}+1\right)=\left(\bar{\varepsilon}^{1}+2, \bar{m}\right)
$$

up to permutation with a suitable sequence of integers $\bar{m}=\left(m_{1}, \ldots\right.$, $\left.m_{a+r_{0}+r_{1}}\right)$. For an arbitrary graded $R$-module $N=\bigoplus_{n} N_{n}$, we denote $\operatorname{dim}_{k}\left(N_{n}\right)$ by $l(N ; n)$.

Lemma (A.3). With the notation above put

$$
\left\{\begin{array}{l}
L(M)=R\left[-\bar{\varepsilon}^{0}-1\right]^{2} \oplus R\left[-\bar{\varepsilon}^{1}\right] \\
L_{1}(B, M)=R\left[-a,-\bar{n}^{1}\right] \oplus L(M)
\end{array}\right.
$$

Then,

$$
\begin{gathered}
l(M ; n)-l\left(R\left[-\bar{\varepsilon}^{0}\right] ; n\right)+l\left(L_{1}(B, M) ; n\right)-l(R[-\bar{m}] ; n) \\
=\left(\begin{array}{c}
n-a+3 \\
3
\end{array}\right)_{+}+\sum_{i=1}^{a}\left(\begin{array}{c}
n-n_{i}+2 \\
2
\end{array}\right)_{+}+\sum_{j=1}^{b}\left(\begin{array}{c}
n-\varepsilon_{j}^{2}+1 \\
1
\end{array}\right)_{+}
\end{gathered}
$$

Proof. Notice that $\left(\begin{array}{c}n+1 \\ m\end{array}\right)_{+}-\left(\begin{array}{c}n \\ m\end{array}\right)_{+}=\left(\begin{array}{c}n \\ m-1\end{array}\right)_{+}$for all integers $n$ and $m \geq 1$. The assertion follows from direct computations. 
Let $\mathscr{L}(M)$ denote the sheaf on $\mathbf{P}^{3}$ associated with $L(M)$ and let $\mathscr{E}(M)$ be the vector bundle of rank $r_{0}+r_{1}$ defined by the exact sequence

(A. 4)

$$
\begin{gathered}
0 \longrightarrow \mathscr{E}(M) \longrightarrow \mathscr{L}(M) \stackrel{\mu}{\longrightarrow} \mathcal{O}_{\mathrm{p}^{3}}\left[-\bar{\varepsilon}^{0}\right] \longrightarrow 0 \\
\mu=\left(x_{1} 1_{r_{0}}-A_{1}, x_{2} 1_{r_{0}}-A_{2}, H\right)
\end{gathered}
$$

where for each $i(i=1,2) \quad A_{i}$ is the matrix with components in $S$ which represents the action of $x_{i}$ on $M$ arising from multiplication (cf. [2; (2.5.3)]). Put
(A. 5)
$\mathscr{F}(B, M)=\mathcal{O}_{\mathbf{p}^{3}}\left(-a ;-\tilde{n}^{1}\right) \oplus \mathscr{E}(M)$.

One sees by (A.2), (A.4) and (A.5) that $c_{1}(\mathscr{F}(B, M))=-\sum_{i=1}^{a+r_{0}+r_{1}} m_{i}$.

Given a global section $\bar{s}=\left(s_{1}, \ldots, s_{a+r_{0}+r_{1}}\right)$ of $\mathscr{F}(B, M) \otimes \mathcal{O}_{\mathrm{p}^{3}}(\bar{m})$, we can therefore construct the complex

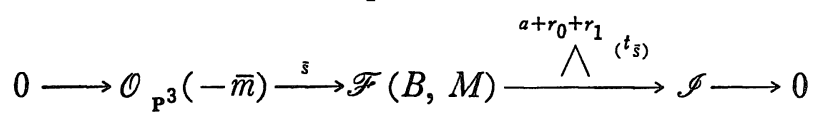

in the usual way, where $\mathscr{I} \subset \mathcal{O}_{\mathbf{p}^{3}}$ and locally $\bar{s}$ is understood to be an $\left(a+r_{0}+r_{1}+1\right) \times\left(a+r_{0}+r_{1}\right)$-matrix.

Proposition (A. 7) (cf. $[2 ; \S 2])$. If $\mathscr{I}$ defines a curve $X$, then $B(X)=B$.

Proof. Note first that (A.6) is exact by hypothesis. We have and

$$
M(X) \cong \bigoplus_{n} H^{1}(\mathscr{F}(B, M)(n)) \cong M
$$

$$
\begin{aligned}
h^{0}(\mathscr{I}(n)) & =h^{0}(\mathscr{F}(B, M)(n))-l(R[-\bar{m}] ; n) \\
& =l\left(L_{1}(B, M) ; n\right)-l\left(R\left[-\bar{\varepsilon}^{0}\right] ; n\right)+l(M ; n)-l(R[-\bar{m}] ; n)
\end{aligned}
$$

by (A. 1), (A.4), (A.5) and (A.6). The assertion therefore follows from (A. 1), (A.3) and the characterization (I. 1), (I. 2), (I.3)' of a basic sequence.

Suppose that $\bar{m}=\left(\bar{m}^{1}, \bar{m}^{2}\right), \bar{s}=\left(\bar{s}^{1}, \bar{s}^{2}\right)$ with $\bar{s}^{i} \in \mathrm{H}^{0}\left(\mathscr{F}(B, M) \otimes \mathcal{O}_{\mathrm{p}^{3}}\left(\bar{m}^{i}\right)\right)$ $(i=1,2)$ and that 


$$
\mathscr{G}:=\operatorname{Coker}\left(\mathcal{O}_{\mathbf{P}^{3}}\left(-\bar{m}^{1}\right) \stackrel{s^{1}}{\longrightarrow} \mathscr{F}(B, M)\right)
$$

is locally free of rank $h$. Then the complex (A.6) can be reduced to (A. 8)

$$
0 \longrightarrow \mathcal{O}_{\mathbf{P}^{3}}\left(-\bar{m}^{2}\right) \stackrel{\bar{s}^{2}}{\longrightarrow} \mathscr{G} \stackrel{\bigwedge^{h-1}\left({ }^{t}\left(\bar{s}^{2}\right)\right)}{\longrightarrow} \mathscr{I} \longrightarrow 0,
$$

which we call a reduction of (A.6). Let $\bar{s}_{1}, \bar{s}_{2}, \ldots, \bar{s}_{e}$ be a basis of $\mathrm{H}^{0}\left(\mathscr{G} \otimes \mathcal{O}_{\mathbf{P}^{3}}\left(\bar{m}^{2}\right)\right), \zeta_{i}(1 \leq i \leq e)$ parameters and $\tilde{s}:=\sum_{i=1}^{e} \zeta_{i} \bar{s}_{i}$ a global section of $\mathscr{G} \otimes_{\mathcal{O}_{\mathbf{P}^{3}}} \mathcal{O}_{\mathbf{P}_{T}^{3}}\left(\bar{m}^{2}\right)$ on $\mathbb{P}_{T}^{3}$, where $T:=\operatorname{Spec} k\left[\zeta_{i} ; 1 \leq i \leq e\right]$. As is well known, if the scheme $\tilde{X} \subset \mathbb{P}_{T}^{3}$ defined locally by the maximal minors of $\tilde{s}$ is of relative dimension 1 over $T$ and smooth over $k$ outside a subscheme of codimension larger than or equal to 4 in $\mathbb{P}_{T}^{3}$, there is a Zariski open set $U \subset T$ such that $\tilde{X} \times_{T}\{u\}$ is a smooth curve for every $u \in U$. Here we present an interesting

Example (A.9). Let $\rho$ be a primitive $a$-th root of unity $(a \geq 2)$ and set

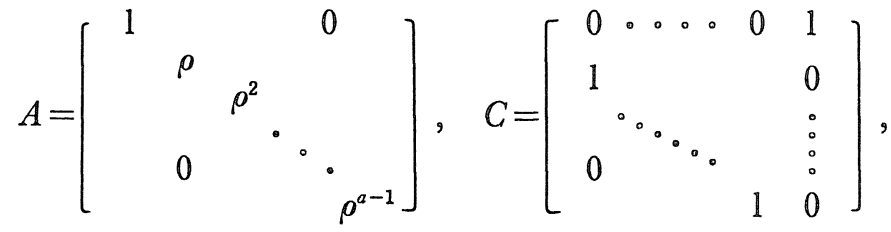

$$
\begin{aligned}
& V_{1}=x_{1} 1_{a}-x_{3} A, V_{2}=x_{2} 1_{a}-x_{4} A, \\
& H_{1}=x_{3} 1_{a}-x_{4} C, H_{2}=x_{4}^{2} 1_{a}, H=\left(H_{1}, H_{2}\right), \mu=\left(V_{1}, V_{2}, H\right), \\
& M=R[-(a-2)]^{a} / \operatorname{Im}^{R}(\mu) .
\end{aligned}
$$

Since $M$ has the minimal free resolution

$$
0 \longrightarrow S\left[-(a+1)^{a}\right] \stackrel{\left(\begin{array}{c}
-H_{2} \\
H_{1}
\end{array}\right)}{\longrightarrow} S\left[-(a-1)^{a},-a^{a}\right] \stackrel{H}{\longrightarrow} S\left[-(a-2)^{a}\right] \rightarrow M \rightarrow 0
$$

as an $S$-module, we have $\bar{\varepsilon}^{0}=(a-2)^{a}, \bar{\varepsilon}^{1}=\left((a-1)^{a}, a^{a}\right), \bar{\varepsilon}^{2}=(a+1)^{a}$. Consider the basic sequence $B=\left(a ;(a+1)^{a} ;(a+1)^{a}\right)$. Since $\bar{m}=\left(a^{3 a}\right.$, $\left.(a+1)^{a}\right)$ and $\mathscr{F}(B, M)=\mathcal{O}_{\mathrm{p}^{3}}\left(-a,-(a+1)^{a}\right) \oplus \mathscr{E}(M) \quad\left(A_{1}=x_{3} A, A_{2}=\right.$ $\left.x_{4} A\right)$, (A. 6) has a reduction with $\bar{m}^{1}=\left(a,(a+1)^{a}\right), \bar{m}^{2}=a^{3 a-1}$ and $\mathscr{G}=\mathscr{E}(M)$. We see that there is a smooth irreducible curve $X$ with $B(X)=B$ and $M(X) \cong M$ by the following facts. The details are left to the interested readers.

1) The basis of $\mathrm{H}^{0}(\mathscr{E}(M)(a))$ is given by the columns of the matrix 


$$
\mu_{1}=\left[\begin{array}{ccc}
-V_{2} & -H_{1} & 0 \\
-V_{1} & 0 & -H_{1} \\
0 & V_{1}+x_{4}[A, C] & V_{2} \\
0 & C[A, C] & {[A, C]}
\end{array}\right],
$$

where [,] denotes the Lie bracket.

2) The divisor $D \subset \mathbf{P}^{3}$ defined by the determinant of the 3 a sections of $\mathscr{E}(M)(a)$ above coincides with the Fermat surface $x_{1}^{a}-x_{2}^{a}-x_{3}^{a}+x_{4}^{a}=0$ and is smooth.

3) This implies that $\mathscr{E}(M)$ is generated over $\mathcal{O}_{\mathbf{p}^{3}}$ by $\mathrm{H}^{0}(\mathscr{E}(M)(a))$ in the outside of $D$ and that the dimension of the vector subspace of $\mathscr{E}(M) \mid{ }_{D} \otimes k(p t)$ generated over $k$ by $\mathrm{H}^{0}(\mathscr{E}(M)(a))$ is $3 a-1$ for each $p t \in D$. The scheme $\tilde{X}$ therefore fulfills the required condition (see the proof of $[11 ;$ Theorem (3.3)]).

Note that the resulting curve lies on $D$.

\section{References}

[1] Amasaki, M., Preparatory Structure Theorem for Ideals Defining Space Curves, Publ. RIMS, Kyoto Univ., 19 (1983), 493-518.

[2] On the Structure of Arithmetically Buchsbaum Curves in $\mathbf{P}_{k}^{3}, P u b l . R I M S$, Kyoto Univ., 20 (1984), 793-837.

[3] Examples of Nonsingular Irreducible Gurves Which Give Reducible Singular Points of $\operatorname{red}\left(\mathrm{H}_{d . g}\right)$, Publ. RIMS, Kyoto Univ., 21 (1985), 761-786.

[4] - Integral arithmetically Buchsbaum curves in $\mathbf{P}^{3}$, preprint RIMS-577 (1987).

[5] Ballico, E. and Ellia, Ph., The Maximal rank conjecture for non-special curves in $\mathbf{P}^{3}$, Invent. Math., 79 (1985), 541-555.

[6] Buchsbaum, D. A. and Eisenbud, D., Some Structure Theorems for Finite Free Resolutions, Advances in Mathematics, 12 (1974), 84-139.

[7] Eisenbud, D. and Goto, S., Linear Free Resolutions and Minimal Multiplicity, Journal of Algebra, 88 (1984), 89-133.

[8] Ellia, Ph., Exemples de courbes de $\mathbf{P}^{3}$ à fibré normal semi-stable, stable, Math. Ann., 264 (1983), 389-396.

[9] Gruson, L. et Peskine, C., Genre des courbes de l'espace projectif, Proceedings, Troms $\phi$, Norwy 1977, Lecture Notes in Mathematics, 687, Springer-Verlag.

[10] Hartshorne, R. and Hirschowitz, A., Smoothing Algebraic Space Curves, Algebraic Geometry, Sitges 1983, Lecture Notes in Mathematics, 1124, Springer-Verlag.

[11] Kleimann, S. L., Geometry on Grassmannians and Application to Splitting Bundles and Smoothing Cycles, Publ. Math. I. H.E.S., 36 (1969), 281-297.

[12] Sernesi, E., On the existence of certain families of curves, Invent. Math., 75 (1984), 25-57. 